\title{
Solar H-alpha features with hot onsets ${ }^{\star} \star \star$ III. Long fibrils in Lyman-alpha and with ALMA
}

\author{
R. J. Rutten ${ }^{1,2}$ \\ ${ }^{1}$ Lingezicht Astrophysics, 't Oosteneind 9, 4158 CA Deil, The Netherlands \\ e-mail: R.J.Rutten@uu.nl \\ 2 National Astronomical Observatory of Japan, 2-21-1 Osawa, Mitaka, 181-8588 Tokyo, Japan \\ Received 4 July 2016 / Accepted 4 October 2016
}

\section{ABSTRACT}

\begin{abstract}
In $\mathrm{H}$-alpha most of the solar surface is covered by dense canopies of long opaque fibrils, but predictions for quiet-Sun observations with ALMA have ignored this fact. Comparison with Ly-alpha suggests that the extraordinary opacity of H-alpha fibrils is caused by hot precursor events. Application of a recipe that assumes momentary Saha-Boltzmann extinction during their hot onset to millimeter wavelengths suggests that ALMA will observe $\mathrm{H}$-alpha-like fibril canopies, not acoustic shocks underneath, and will yield data more interesting than if these canopies were transparent.
\end{abstract}

Key words. Sun: chromosphere - Sun: infrared

\section{Introduction}

The Sun consists largely of hydrogen. The diagnostics provided by hydrogen are therefore of prime interest in studies of the solar atmosphere, in particular the chromosphere. Here I discuss the principal ones, Ly $\alpha$ at $1216 \AA, \mathrm{H} \alpha$ at $6563 \AA$, and the millimeter continua accessible to the Atacama Large Millimeter/submillimeter Array (ALMA, website) by estimating and comparing the visibilities of chromospheric features in these diverse spectral windows.

The key observation prompting this study is the well-known fact that in the center of $\mathrm{H} \alpha$ most of the solar surface is covered by opaque slender fibrils, not only for active regions as in Fig. 2 below but also in quieter areas (e.g., Figs. 6-9 of Rutten 2007) and even (as so-called mottles) in very quiet areas as in Fig. 1 of Rouppe van der Voort et al. (2007), except for relatively rare super-quiet locations (upper-left corner in Fig. 1 of Rouppe van der Voort et al. 2007, lower-left corner in Fig. 9 of Rutten 2007).

The question addressed here is how these fibrils will appear in ALMA images. My prediction: even more opaque.

The key premise of this study is that the $\mathrm{H} \alpha$ fibril canopies stem from propagating heating events (PHE) that cause a wideranging family of features emanating from magnetic concentrations in network and plage. The most familiar members of this family are off-limb spicules-II (De Pontieu et al. 2007b,c) and their on-disk representations as rapid blue-wing excursions in $\mathrm{H} \alpha$ (RBE, Langangen et al. 2008; Rouppe van der Voort et al. 2009; Sekse et al. 2012) and similar rapid red-wing excursions in $\mathrm{H} \alpha$ (RRE, Sekse et al. 2013), although their drivers remain unidentified (Pereira et al. 2012). These must be more energetic than simple acoustic shocks running up slanted

\footnotetext{
$\star$ An additional file is available at the end of this article.

$\star \star$ This study is offered as compliment to M.W.M. de Graauw. Our ways, objects, instruments and spectral domains parted after the 1970 eclipse but converge here.
}

fluxtubes because the latter produce well-understood dynamic fibrils around plage and active network (Hansteen et al. 2006; De Pontieu et al. 2007a) and similar but shorter dynamic fibrils in active regions (Rouppe van der Voort \& de la Cruz Rodríguez 2013).

I postulate below that there must be more horizontal PHEs that produce the observed ubiquitous long $\mathrm{H} \alpha$ fibrils. My working hypothesis is that the latter are post-PHE contrails, comparable to the contrails drawn by passing aircraft on our sky.

The second study in this series describes an example of such a solar contrail: a large dark $\mathrm{H} \alpha$ fibril marking the earlier passage of a spectacular PHE (Rutten \& Rouppe van der Voort 2017, henceforth Pub II). The PHE shared properties with more ordinary RBEs but it was unusually large and energetic and was launched in a more horizontal direction. Minutes later the large $\mathrm{H} \alpha$ fibril appeared as if the PHE trajectory had become outlined with a fat marker pen.

Such delayed $\mathrm{H} \alpha$ contrail formation stems from the enormous relative abundance of hydrogen and the large excitation energy of its first excited level $(n=2$ at $10.2 \mathrm{eV}$ ). These properties produce particular formation characteristics that affect all three spectral windows: slow nonequilibrium ionization/recombination balancing at low temperature, Saha-Boltzmann or near-Saha-Boltzmann high-level and ion populations at high temperature and density, large extinction at high temperature that remains large in cool PHE aftermaths.

These extraordinary characteristics are elaborated in Sect. 2 with reference to and in sequel to the first study in this series (Rutten 2016, henceforth Pub I). It formulated a hot-onset recipe to understand the diverse visibilities of Ellerman bombs (photospheric reconnection events; review by Rutten et al. 2013). In brief, the recipe is to evaluate the extinction of $\mathrm{H} \alpha$ at the onset of a hot event assuming Saha-Boltzmann population of the hydrogen $n=2$ level and to sustain this high value during subsequent cooling, also more widely around it. I suggest here that 
this post-Saha-Boltzmann-extinction (PSBE) recipe also applies to the long fibrils constituting the $\mathrm{H} \alpha$ canopies.

Section 3 compares solar $\mathrm{H} \alpha$ and $\operatorname{Ly} \alpha$ images as major motivation to invoke PHE's as fibril generator. Additional evidence comes from the paucity of internetwork shock scenes in $\mathrm{H} \alpha$ and fibril incongruity between $\mathrm{H} \alpha$ and Ca II $8542 \AA$ (Sect. 4).

Section 4 also discusses how to detect these PHEs, why numerical simulations so far fail to reproduce the actual $\mathrm{H} \alpha$ chromosphere, and what fibril widths and temperatures ALMA will measure.

The conclusion (Sect. 5) summarizes this study by predicting that in ALMA images the Sun will appear as the fibrilar $\mathrm{H} \alpha$ chromosphere, largely obscuring the shock-interference internetwork scenes that have been extensively foretold in quiet-Sun predictions for ALMA based on numerical simulations (details, reviews and references in Wedemeyer et al. 2016a,b).

\section{Extinction in and and after hot precursors}

\subsection{PSBE recipe}

Pub I based the PSBE recipe for hot-onset features on the one-dimensional plane-parallel hydrostatic model atmosphere of Avrett \& Loeser (2008) and the two-dimensional nonequilibrium MHD simulation of Leenaarts et al. (2007), regarding these as the atmospheres of hypothetical stars called ALC7 and HION. They share properties with the Sun: in the case of ALC7 the emergent disk-center average spectrum, in the case of HION the presence of granulation, sound waves, acoustic internetwork shocks, network-like magnetic fields, and even dynamic fibrils. In my opinion HION comes much closer to the actual Sun even while being only two-dimensional, whereas ALC7 is a superb didactic star of which the detailed spectrum is easily synthesized with the splendid virtue of being fully understandable ${ }^{1}$.

The ALC7 and HION properties inspiring the PSBE recipe for $\mathrm{H} \alpha$ features are:

- wherever the Ly $\alpha$ source function $S$ equals the Planck function $B(T)$ for temperature $T$ and hydrogen is predominantly neutral the extinction coefficient of $\mathrm{H} \alpha$ is given by the Saha-Boltzmann (henceforth SB) value because the H I ground level is then the population reservoir with NLTE population departure coefficient $b_{1} \approx 1$ and $S \approx\left(b_{2} / b_{1}\right) B$ (Sect. 2.1 of Pub I);

- although $\operatorname{Ly} \alpha$ is the most scattering line in the solar spectrum it thermalizes in the ALC7 chromosphere. The thermalization length for its Doppler core in optical path units is $\Lambda_{\tau}^{\mathrm{D}}=\sqrt{\pi} / \varepsilon$, with $\varepsilon$ the collisional destruction probability per line photon extinction (Avrett 1965). This relation holds for an isothermal constant- $\varepsilon$ atmosphere, but it is a good approximation for the ALC7 chromosphere which is nearly isothermal and has near-constant $\varepsilon \approx 10^{-6}$ because increasing hydrogen ionization compensates the density decrease (display 2: ALC7 model). The very small $\varepsilon$ implies thermalization over a million photon path lengths in ALC7, the most of all chromospheric lines (display 18: Ly $\alpha$ ). However, even such huge scattering extent remains geometrically small because the Ly $\alpha$ line-center extinction coefficient $\alpha$ is also huge and the corresponding photon mean free path $1 / \alpha$ very small, so that $\Lambda_{x}^{\mathrm{D}}=\sqrt{\pi} /(\alpha \varepsilon)$ increases from only $1 \mathrm{~km}$ at the bottom of the ALC7 chromosphere $(h=800 \mathrm{~km})$

\footnotetext{
1 For students of my courses. Recently I added ALC7 line formation diagrams to my example displays when invited to teach at the NAOJ. Some are referenced at the end of this article.
}

to $100 \mathrm{~km}$ at $h=1400 \mathrm{~km}$ where $\mathrm{H} \alpha$ escapes (display 19: $\mathrm{H} \alpha$ ). This estimate is for the Doppler core only. At larger density the Stark wings of Ly $\alpha$ become more important, including partially coherent scattering, and increase the actual thermalization length $\Lambda_{x}$ over which the profile-summed and angle-averaged radiation $\bar{J}$ saturates to the local $B$. The wingescape increase is beyond analytic formulation; numerical tests with the RH code of Uitenbroek (2001) showed that $\Lambda_{x}=10-100 \mathrm{~km}$ in the lower ALC7 chromosphere. This relatively small range implies that the Ly $\alpha$ radiation remains locked-in within it and thermalizes to the local temperature where $\mathrm{H} \alpha$ escapes, producing $S \approx B$ equality in Ly $\alpha$ and SB extinction for $\mathrm{H} \alpha$ (Fig. 1 of Pub I; display 19: $\mathrm{H} \alpha$ );

- shocks in the HION atmosphere have temperatures of order $7000 \mathrm{~K}$ and electron densities around $10^{11} \mathrm{~cm}^{-3}$, just as the ALC7 chromosphere which has $T \approx 6700 \mathrm{~K}$ and $N_{\mathrm{e}} \approx$ $10^{11} \mathrm{~cm}^{-3}$, both nearly constant (display 2: ALC7 model). This equality is not surprising because the emphasis in the ALC7 best-fit construction was on the quiet-Sun ultraviolet spectrum in which non-linear Wien $\mathrm{d} B / \mathrm{d} T$ sensitivity gives larger weight to higher temperature (Carlsson \& Stein 1994) while in quiet areas acoustic shocks dominate the internetwork sub-canopy domain (review in Rutten 1995) so that ultraviolet spectrum fitting replicates the shock temperature spikes (Carlsson \& Stein 1995);

- also within the HION shocks the HI $n=2$ population saturated to the SB value (bottom panels of Fig. 2 of Leenaarts et al. 2007). In this simulation the net photon rates in Lyman transitions were put to zero for tractability following Carlsson \& Stein (2002). This simplification produced high-lying green fibril-like arches in the last panel of Fig. 1 of Leenaarts et al. (2007) which are artifacts, but it does not affect lower layers where radiative Lyman balancing is a good approximation as shown for 1D static models by Vernazza et al. (1981) and for HION-like shocks by Carlsson \& Stein (2002);

- in the HION shocks hydrogen reaches about $10 \%$ ionization with $b_{\mathrm{c}} \approx 0.1$; they would reach full ionization if LTE were valid (thin curves in the second row of Fig. 2 of Leenaarts et al. 2007). Similar NLTE underionization occurs through the ALC7 chromosphere (display 4: ALC7 hydrogen). It does not represent a transition from SB to coronal-equilibrium (CE) partitioning, which in the ALC7 atmosphere occurs at much lower density in the transition region. Instead, it follows the Balmer continuum which is the main hydrogen ionization agent in the chromosphere (e.g., Vernazza et al. 1981; Carlsson \& Stein 2002) and has constant radiation temperature near $5250 \mathrm{~K}$ as a scattering average of photons created in the granulation (display 4: ALC7 hydrogen). In the HION and ALC7 atmospheres the top of the hydrogen atom behaves as a $3.4 \mathrm{eV}$ alkali atom with $n=2$ as ground level having Ly $\alpha$-defined constraining population and with the continuum population at an offset from it that is given by balancing the $B_{\text {Bacont }}(5250 \mathrm{~K}) / B_{\text {Bacont }}(T)$ NLTE ionization driving with photon losses in Balmer and higher lines that govern NLTE recombination (Fig. 3 of Rutten \& Carlsson 1994);

- in the HION atmosphere drastic cooling occurs after a shock has passed. However, in this non-equilibrium (non-E) star the H I $n=2$ population did not adapt instantaneously. Collisional bound-bound balancing has Boltzmann temperature sensitivity through the collisional excitation rate $n_{1} C_{12}$ (p. 50 
and 51 of Rutten 2003) making the $10.2 \mathrm{eV}$ Ly $\alpha$ jump too large for fast thermal balancing at low temperature, as demonstrated by Carlsson \& Stein (2002). In shocks the collisional balancing is fast so that the $\operatorname{Ly} \alpha$ radiation and corresponding $n=2$ population reach the high LTE values, but they then hang near these while the gas cools until the next shock passes (bottom panels of Fig. 2 of Leenaarts et al. 2007). The Balmer continuum and lines couple the ionization degree to this retarded non-E $n=2$ behavior without further retardation, initially giving $b_{\text {cont }} / b_{2} \approx 0.1$ and then reversing to $b_{\text {cont }} / b_{2} \gg 1$ while the gas cools well below $5250 \mathrm{~K}$. The bottom row of Fig. 1 of Leenaarts et al. (2007) shows that the post-shock HION clouds so reach huge overpopulations: up to $b_{2}=10^{12}$ and $b_{\text {cont }}=10^{15}$;

- hot features embedded in cool gas irradiate their surroundings in $\operatorname{Ly} \alpha$ over hundreds of kilometers due to smaller $\varepsilon$ in the cool gas. This radiation boosts $J$ and therefore $S$ in Ly $\alpha$ and with it the $\mathrm{H} \alpha$ extinction across such scattering extents towards the high-temperature values within the feature (Fig. 3 of Pub I; display 19: H $\alpha$; display 26: aureole boosting). A momentary heating event has similar boost-spreading as long as hydrogen remains partially neutral. This then lasts minutes after its occurrence.

With these, the PSBE recipe for $\mathrm{H} \alpha$ features became: (1) evaluate the $\mathrm{H} \alpha$ extinction coefficient during the hot onset of a dynamic feature by assuming the SB value; (2) use the resulting large population also for cooler surrounding gas in reach of scattering Ly $\alpha$ radiation; and (3) maintain this large population subsequently during cooling aftermaths. Pub I so explained the diverse and even discordant visibilities of Ellerman bombs that cannot be reproduced with static equilibrium models.

A comment concerning ALMA: $\mathrm{H} \alpha$-like memory of hot instances in the recent past holds also for hydrogen free-free continua since these are similarly extinction-boosted by retarded hydrogen recombination in cool post-hot episodes, i.e., cool gas where a heating event passed shortly before. Below I concentrate on long $\mathrm{H} \alpha$ fibrils and my interpretation of these as post-hot contrails, but similar non-E boosting must take place in decidedly post-hot phenomena as spicules-II and coronal rain to which I return in the conclusion.

A comment concerning Ly $\alpha$ : its extinction does not suffer from retarded collisional balancing after HION shocks because at $10 \%$ ionization the $\mathrm{H}$ I ground level remains the population reservoir in these. However, it might when recombination follows on hotter precursor events that ionize hydrogen more completely.

\subsection{Saha-Boltzmann extinction}

Figure 1 shows monochromatic extinction coefficients (large panel per pair) and hydrogen and electron densities (small panel per pair) in a parcel of solar gas with given total hydrogen density as function of temperature, assuming SB population partitioning. It was made, similarly to Fig. 5 in Pub I, with IDL programs (on my website) based on LTE programs from a github repository of $\mathrm{A}$. Asensio Ramos that were written by Sánchez Almeida $(1992,1997)$ and were partly based on Wittmann (1974) following Mihalas (1967). I renewed and extended these programs with data and routines in the SolarSoft CHIANTI package (e.g., Dere et al. 1997, Landi et al. 2013). The dotted comparison curves for coronal equilibrium (CE) in the small density panels are directly from CHIANTI.
The horizontal line at $y=-7$ in each extinction panel specifies optical thickness unity for a line of sight crossing a 100-km wide slab. Extinction above this line implies optically thick sampling of a feature of this size.

The extinction curve patterns with their initial rises (except Ly $\alpha$ ), peaks, and steep to slow declines for increasing temperature are primarily set by hydrogen ionization, in which the crossover from atoms to protons shifts left and steepens from row to row. The extinction patterns change correspondingly but retain their qualitative offset and cross-over behavior. The main changes are the decreases $\propto N_{\mathrm{H}}$ or $\propto N_{\mathrm{H}} N_{\mathrm{e}}$ from row to row, most clearly exhibited by the curve separations from the $y=-7$ thickness indicator line, and the H I free-free extinction increase $\propto \lambda^{2}$ along rows (Eq. (5.19a) of Rybicki \& Lightman 1986; Eq. (2.79) of Rutten 2003).

The $\alpha_{\mathrm{ff}} \propto \lambda^{2}$ increase shifts the $\tau=1$ height of radiation escape across the ALC7 chromosphere. At $0.35 \mathrm{~mm}$ it lies at its bottom, at $3 \mathrm{~mm}$ at its top. Because the rows of Fig. 1 cover this range in gas density and the ALC7 temperature is nearly constant at $6700 \mathrm{~K}$, the corresponding SB extinction coefficients can be read off as $6700 \mathrm{~K}$ samplings of the three diagonal (first-to-last) panels of Fig. 1. The HION shocks have total hydrogen density $N_{\mathrm{H}} \approx 10^{14}$, between the first two rows.

The actual hydrogen ionization follows cooler Balmer radiation as noted above, but this NLTE departure produces only slight desteepening of the steep increases in the HIff curves around the $T=5250 \mathrm{~K}$ pivot. For ALC7 it represents only a one dex tilt correction of the 10 dex increase. For HION it amounts to correction by +3 dex at $3000 \mathrm{~K}$ in the shock aftermaths diminishing to -1 dex at $7000 \mathrm{~K}$ in the shocks themselves, still relatively small.

I added the dotted CE curves to the density panels to indicate hydrogen ionization at lower gas densities. They are unrealistic at chromospheric densities but they do show the trend because from very high to very low density the truth shifts from SB to $\mathrm{CE}$. The invariant $\mathrm{CE}$ curve cross-over at $16000 \mathrm{~K}$ implies that at decreasing density the leftward shift and steepening of the SB hydrogen cross-overs get compensated.

I now discuss the spectral features in Fig. 1 one by one.

Ly $\alpha$ is of course the extinction champion at low temperature. Since its lower level then contains virtually all hydrogen SB extinction is guaranteed. At higher temperatures its extinctioncoefficient decline follows the neutral-hydrogen density decline. In $\mathrm{CE}$ this is much less steep than for SB, predicting highertemperature $\operatorname{Ly} \alpha$ presence for lower densities. A solar feature needs to be only kilometer-size to get optically thick in this line. Only above $40000 \mathrm{~K} \mathrm{H} \alpha$ obtains larger SB extinction from the $g_{2} / g_{1}=8$ statistical-weight ratio. At lower temperature anything opaque in $\mathrm{H} \alpha$ is necessarily much more opaque in Ly $\alpha$.

For $\mathrm{H} \alpha$ the SB extinction assumption is correct when hydrogen ionization at high density increases $\varepsilon$ in Ly $\alpha$ sufficiently within opaque features. As noted above $b_{2} \approx b_{1} \approx 1$ indeed holds throughout the ALC7 chromosphere and also within the HION shocks. At higher temperature the corresponding increase in $\varepsilon$ produces Ly $\alpha$ thermalization within yet smaller features.

For Ca II $8542 \AA$ the assumption of SB extinction is also reasonable, actually an underestimate where photon losses in the Ca II infrared lines cause NLTE overpopulation of their lower levels (Fig. 1 of Pub I, display 15: Ca II $8542 \AA$ ). Observationally, this line shows fibrils comparably to $\mathrm{H} \alpha$ near network but not further out from network, an important incongruity to which I return in Sect. 4. 

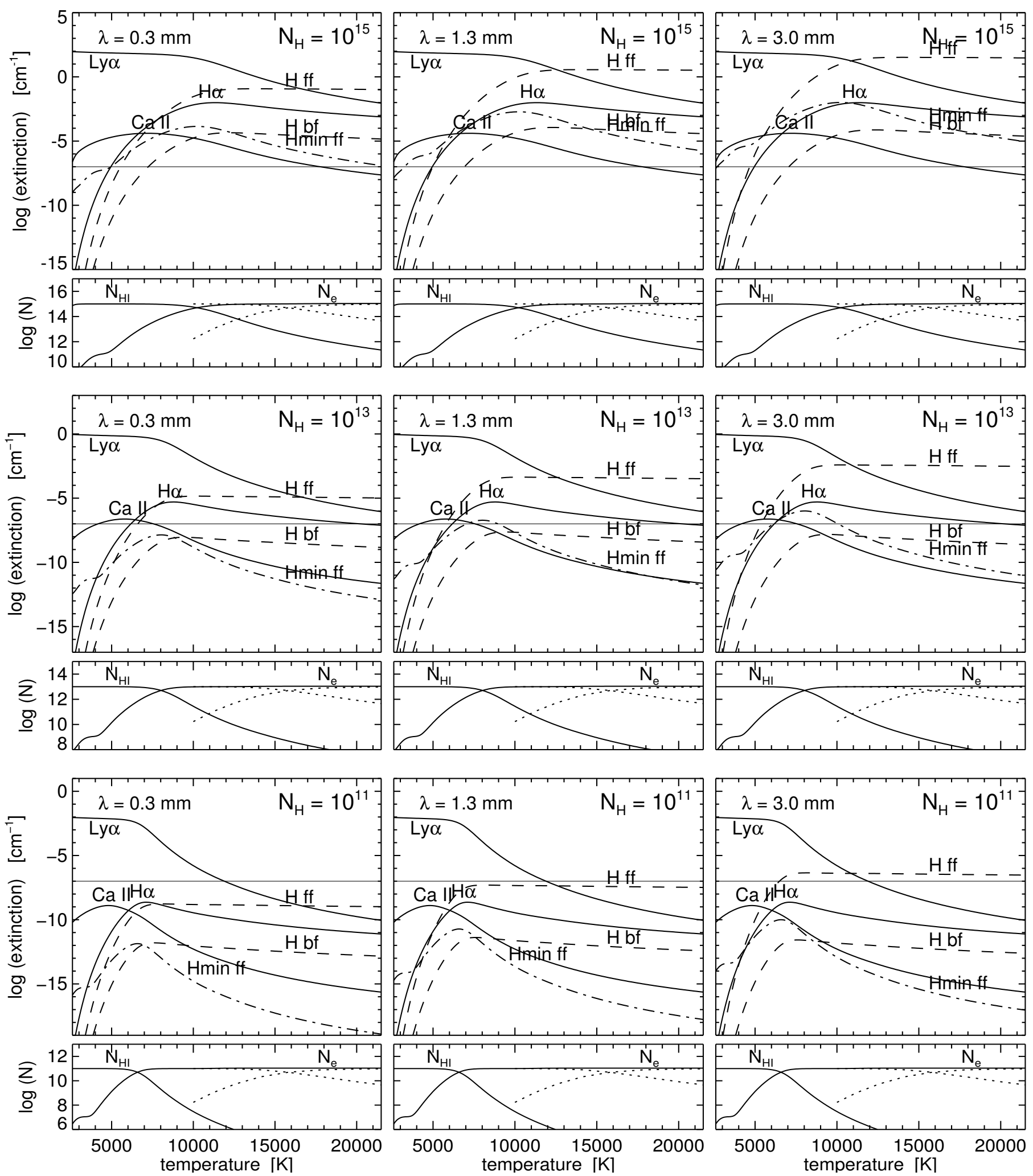

Fig. 1. Saha-Boltzmann (SB) line extinction coefficient against temperature at the centers of Ly $\alpha, \mathrm{H} \alpha$ and Ca II $8542 \AA$ (solid) and SB continuous extinction coefficient of the $\mathrm{H}$ I free-free and bound-free contributions (dashed) and the $\mathrm{H}^{-}$free-free contribution (dot-dashed) at three ALMA wavelengths (from left to right $0.35,1.3$ and $3.0 \mathrm{~mm}$ ), for gas of solar composition with different total hydrogen densities (from top to bottom $N_{\mathrm{H}}=10^{15}, 10^{13}$ and $10^{11} \mathrm{~cm}^{-3}$, corresponding to the bottom, middle and top of the ALC7 chromosphere). The horizontal line at $y=-7$ specifies optical thickness unity for a line of sight through a $100 \mathrm{~km}$ wide slab. The small panels underneath each extinction graph show the competing neutral hydrogen and electron densities (solid, particles $\mathrm{cm}^{-3}$ ). The overlaid dotted curves starting at $10000 \mathrm{~K}$ are coronal-equilibrium (CE) neutral hydrogen and proton densities from CHIANTI. The density scales have the same logarithmic unit (dex) size as the extinction scales to enable slope comparisons. The $y$-axis scales shift between rows for better curve visibility, 
$\mathrm{H}$ I free-free extinction behaves remarkably similar to $\mathrm{H} \alpha$ in its steep initial increases. Beyond these this contribution dominates the ALMA continua and grows steeply $\propto \lambda^{2}$ while decreasing slowly $\propto T^{-3 / 2}$ across these parameter ranges. The $\mathrm{H} \mathrm{I} \mathrm{ff} \mathrm{/} \mathrm{H} \alpha$ extinction coefficient ratio increases with both to very large values. Anything opaque in $\mathrm{H} \alpha$ will be at least similarly opaque at $\mathrm{mm}$ wavelengths. Anything hot and opaque in $\mathrm{H} \alpha$ will be much more opaque at the longer ALMA wavelengths, even exceeding Ly $\alpha$ above $12000 \mathrm{~K}$.

$\mathrm{H}$ I bound-free extinction is less important than $\mathrm{H}$ I free-free extinction in all panels.

$\mathrm{H}$-minus free-free extinction is only important below about $5000 \mathrm{~K}$ where the small plateau in the first panel and in the electron density curves illustrates that metal ionization rather than hydrogen ionization governs the optical and infrared continua from the photosphere. The upper photosphere is fully transparent in $\mathrm{H} \alpha$ but not at mm wavelengths.

The extinction coefficients of Thomson and Rayleigh scattering were also evaluated, but they are not shown because they are not competitive in this parameter domain.

\subsection{Non-equilibrium extinction}

The curves in Fig. 1 are for instantaneous LTE but permit interpretation of the non-E results of the HION simulation. While Ly $\alpha$ thermalizes within the HION shocks giving SB extinction to $\mathrm{H} \alpha$, during the post-shock cooling the gas temperature follows the very steep Boltzmann slopes left of the $\mathrm{H} \alpha$ peaks in Fig. 1 and so the $\mathrm{H} \alpha$ extinction would get far smaller, over 10 orders of magnitude, if SB remained valid. However, this is not the case: retarded Ly $\alpha$ settling maintains the high $n=2$ population reached in the shocks and so produces the huge $b_{2}$ values in the last panels of Figs. 1 and 2 of Leenaarts et al. (2007). These are called "NLTE overpopulations" but a better name would be "SB underestimates". In the HION shock aftermaths the temperature drops typically from $7000 \mathrm{~K}$ to $3000 \mathrm{~K}$, indeed corresponding to $10 \mathrm{dex}$ SB underestimate for $n=2$ due to the very steep Wien $\mathrm{d} B / \mathrm{d} T$ at $\operatorname{Ly} \alpha$. The much larger actual population therefore gives $\mathrm{H} \alpha$ PSBE formation in HION post-shock cooling clouds. Figure 1 suggests that such time-lagged PSBE boosting may likewise occur in $\mathrm{H}$ I ff continua, with large boosts at longer wavelengths.

\subsection{Source functions and intensities}

ALMA is correctly advertised as "linear thermometer" for optically thick structures in the solar atmosphere. The mmwavelength $S=B=\left(2 c k / \lambda^{4}\right) T$ equality indeed holds wherever the kinetic Maxwell distribution holds since free-free transitions are always collisional, free-free extinction dominates over the whole parameter domain of Fig. 1, and the Rayleigh-Jeans limit applies very well. The advertising might add that the LTE nature of the source function guarantees sharp detail rendition without blurring from scattering within the solar atmosphere, i.e., that the image resolution is limited only by ALMA itself.

The numerical thermometer demonstration in Fig. 4 of Wedemeyer (2016), copied from Fig. 1 of Wedemeyer-Böhm (2007), is therefore just an elaborate demonstration of the simple Eddington-Barbier approximation for the observed intensity $I \approx S\left(\tau_{\mu}=1\right)=B\left(\tau_{\mu}=1\right) \propto T\left(\tau_{\mu}=1\right)$ with $\tau_{\mu}$ the summed extinction along the line of sight. A much earlier and more elegant demonstration was the careful labeling $S=B$ added by E.H. Avrett to all continuum-formation displays for wavelengths above $1.6 \mu \mathrm{m}$ in the wonderful Fig. 36 of Vernazza et al. (1981).
The issue in interpreting solar observations from ALMA is therefore not a source function issue but an extinction issue. What is the thermometer sampling? More precisely: is the extinction defining the $\tau_{\mu}=1$ depth in optically thick features given by the present or by the past? In the first case the opacity of features in ALMA images is regulated instantaneously and classical hydrogen ionization modeling assuming instantaneous statistical equilibrium suffices. In the second case the feature opacities depend on what happened before and the modeling must be non-E including gas histories even in chromospheric conditions (cf. Kneer \& Nakagawa 1976; Klein et al. 1976; Joselyn et al. 1979; Poletto 1979; Kneer 1980; Carlsson \& Stein 2002; Leenaarts \& Wedemeyer-Böhm 2006; Wedemeyer-Böhm et al. 2007; Leenaarts et al. 2007, and references in Sect. 4.4).

In contrast, $\operatorname{Ly} \alpha$ has instantaneous extinction wherever hydrogen is mostly neutral but it is the quintessential two-level scattering line in the solar spectrum with very small $\varepsilon$. Any sizable feature will harbor the well-known scattering decline from $S \approx B$ inside to very low $S \approx \sqrt{\varepsilon} B$ at its surface (Fig. 1ff of Avrett 1965; Mihalas 1970; Sect. 4.3 of Rutten 2003), unless it has steeply outward increasing temperature near its surface along the line of sight (as when that is the transition to the corona).

$\mathrm{H} \alpha$ has been regarded as "photo-electrically controlled" since Thomas (1957) and Jefferies \& Thomas (1959), meaning that the multi-level detour terms specified by $\eta$ in the general breakdown of the line source function $S^{l}=(1-\varepsilon-\eta) \bar{J}+\varepsilon B+\eta S^{\mathrm{D}}$ dominate over the resonance scattering terms set by $\varepsilon$. However, in standard model atmospheres such as ALC7 this is not correct for the chromospheric layers: there even $\mathrm{H} \alpha$ is primarily a twolevel scattering line with $S^{l} \approx \bar{J}$ (Fig. 12 of Rutten \& Uitenbroek 2012; display 19: $\mathrm{H} \alpha$ ).

The upshot is that for optically thick features darkness (low brightness temperature) in ALMA images indeed means low gas temperature around the Eddington-Barbier depth $\tau_{\mu}=1$, with sharp rendering of detail since there is no scattering. In contrast, at the centers of $\operatorname{Ly} \alpha$ and $\mathrm{H} \alpha$ darkness generally does not suggest low temperature but large opacity bringing $\tau_{\mu}=1$ further out along the scattering decline, with blurring from resonance scattering.

Similarly, at mm wavelengths bright optically thick features directly imply correspondingly high temperature around $\tau_{\mu}=1$ with sharp rendering, but in scattering line cores brightness stems from much deeper-sited heating or a very steep negative $T\left(\tau_{\mu}\right)$ gradient or domination by recombination detours, again with blurring from scattering.

\subsection{Conclusion of this section}

Hot precursor events such as the shocks in the HION atmosphere have large PSBE opacity in $\mathrm{H} \alpha$ during the subsequent cooling phase. Similar or larger post-hot extinction is expected at $\mathrm{mm}$ wavelengths. For hotter onset features much more PSBE opacity is expected, with increasing boosts at longer wavelengths. Scattering blurs such events in Ly $\alpha$ and $\mathrm{H} \alpha$ but not in mm continua.

\section{Ly $\alpha$ versus $\mathrm{H} \alpha$}

Figure 2 compares solar active-region scenes in the core of $\mathrm{H} \alpha$ and wide-band Ly $\alpha$. Regretfully, they are far from simultaneous or cospatial, but they do exhibit comparable solar scenes.

Why they differ so much has long puzzled me. Anything visible in $\mathrm{H} \alpha$ has much larger opacity in $\operatorname{Ly} \alpha$ so that any scene 


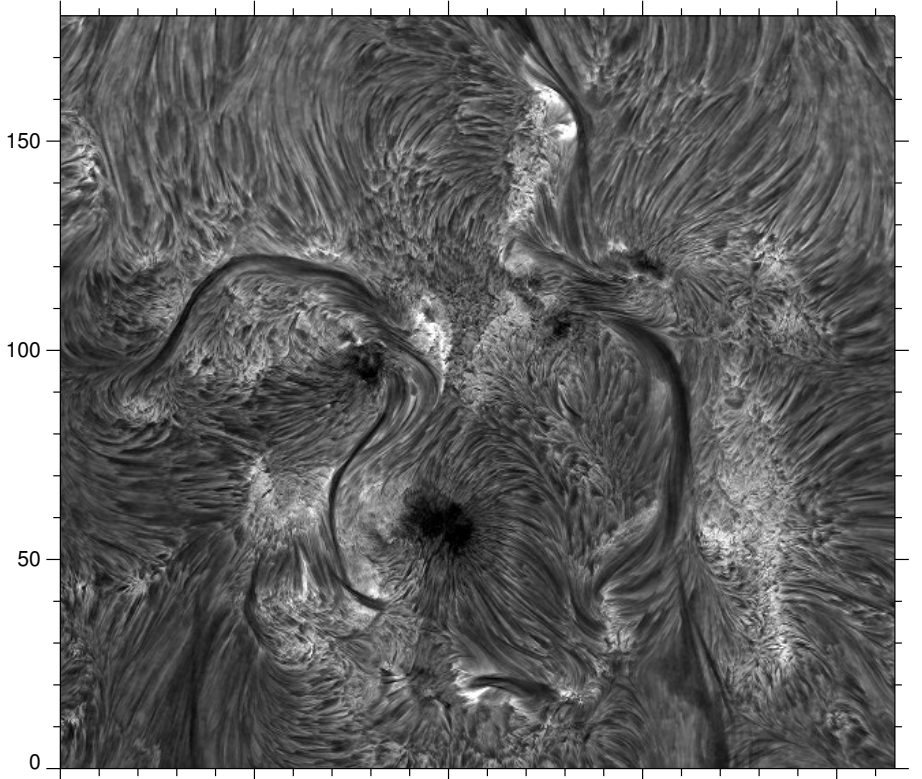

$50 \quad 100$

150

200

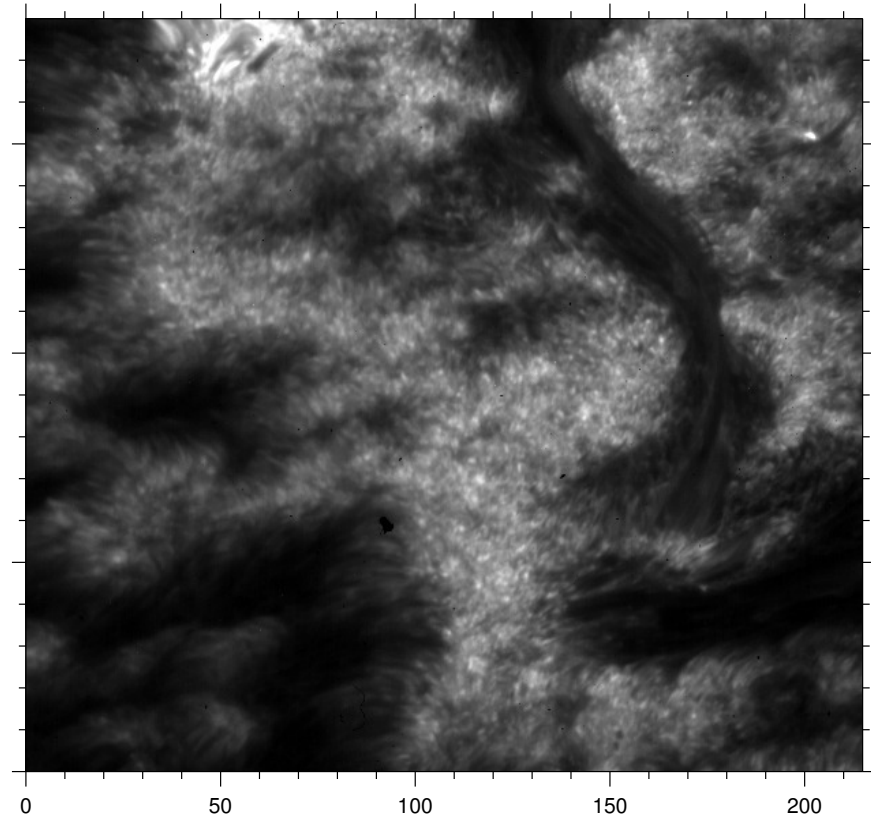

Fig. 2. Comparison of solar scenes in $\mathrm{H} \alpha$ and $\mathrm{Ly} \alpha$. Scales in arcsec. The images have identical field size but sample the Sun at different times (July 8, 2005 and June 14, 2002) and viewing angles $(\mu=0.98$ and $\mu=0.68$ with the limb to the top). The first is an H $\alpha$ line-center mosaic constructed by P. Sütterlin from nine images he took with the Dutch Open Telescope (DOT, Rutten et al. 2004). The second is part of the 13th wide-band Ly $\alpha$ image from VAULT-II (Vourlidas et al. 2010). The DOT pixels were 0.071 arcsec, the VAULT pixels 0.124 arcsec. The claimed resolution is 0.3 arcsec for both, but zoom-in per pdf viewer suggests that the DOT image comes closer to this value than the VAULT image.

observed in $\mathrm{H} \alpha$ should be exaggerated in Ly $\alpha$. However, the scenes in Fig. 2 are very dissimilar.

Both lines are strongly resonant scattering. For $\mathrm{H} \alpha$ it implies lower intensity at larger opacity from sampling the outward scattering decline further out. For Ly $\alpha$ larger opacity likewise produces a deeper self-absorption dip at line center, but the VAULT images sum the full profile and are dominated by the profile peak heights and widths. The peaks scatter independently due to partial redistribution and increase at higher temperature. One so expects to see bright $\operatorname{Ly} \alpha$ features from hot sheaths around cooler structures that show up relatively dark in $\mathrm{H} \alpha$. In particular, one would expect to recognize long dark $\mathrm{H} \alpha$ fibrils as extended filamentary bright transition-region sheets along them (Rutten 2007).

This expectation does hold for dynamic fibrils in the VAULTII near-limb images (Koza et al. 2009), but the VAULT-II disk image in Fig. 2 does not show long fibrils. The long active-region filaments present in both images appear similar, by chance even in shape, except for wider width in $\operatorname{Ly} \alpha$ that one indeed expects from much larger opacity at given neutral hydrogen density. The "mossy" activity areas in $\mathrm{H} \alpha$ harboring bright grains also seem to have similar counterparts in $\operatorname{Ly} \alpha$, but such areas are much more wide-spread in Ly $\alpha$ whereas the Ly $\alpha$ scene lacks the domination by long fibrils observed in $\mathrm{H} \alpha$ everywhere around the mossy areas. There appears to be no bright transition-regionsheet mapping of long $\mathrm{H} \alpha$ fibrils in $\mathrm{Ly} \alpha$, contrary to my earlier expectation.

The Ly $\alpha$ scene does contain grayish fibrilar features pointing away from active areas, e.g., spreading left from the active region in the lower-left corner of Fig. 2 above $x=100$. Above $x=50$ there are fans of such features diverging leftward from more concentrated gray patches presumably at active network. Such fibrilar features were called "loop-like structures" by Patsourakos et al. (2007) and "threads" by e.g., Judge \& Centeno (2008); the latter type of fans were called "comet heads" by Judge \& Centeno (2008). These Ly $\alpha$ fibrils measure about 10 arcsec, much shorter than long $\mathrm{H} \alpha$ fibrils.

In summary, while the $\mathrm{H} \alpha$ internetwork scene is dominated by long fibrils, Ly $\alpha$ shows only short fibrils jutting out from activity. I can only reconcile this striking difference by postulating that Ly $\alpha$ primarily shows hot events while $\mathrm{H} \alpha$ fibrils show subsequent cooler aftermaths.

I therefore suggest that bright Ly $\alpha$ grains represent initial PHEs with steep source function increases and with more horizontal field-aligned launching at the edges of activity areas following magnetic canopy expansions over adjacent internetwork. Indeed, when blinking successive co-aligned VAULT-II images one observes substantial proper motion for some. Note that Ly $\alpha$ scattering gives them extents of order 0.5 arcsec even if the actual PHE was smaller.

Figure 2 then suggests that the more horizontal PHEs leave cooling gas producing $\mathrm{H} \alpha$ fibrils as in the example of Pub II. Such gas necessarily has much larger opacity in $\operatorname{Ly} \alpha$ than in $\mathrm{H} \alpha$, but with much smaller opacity contrasts. In $\mathrm{H} \alpha$ adjacent fibrils sample different histories, mutually out of phase with each coming down the steep decline in Fig. 1 to lower temperature at a few minutes of retardation after its individual precursor event. The gas densities in pressure-equilibriated cooling clouds may not differ much, but their histories and the resulting $\mathrm{H} \alpha$ opacities that define their brightness contrasts while they remain optically thick can differ very much from one to another. In contrast, wherever hydrogen is predominantly neutral the Ly $\alpha$ opacity varies only with the local gas density and hence differs much less between adjacent fibrils.

The Ly $\alpha$ source function follows the Ly $\alpha$ radiation $J$. Even while this remains near its high precursor value from being boxed-in where $\mathrm{H} \alpha$ escapes inside Ly $\alpha$-thick cooling clouds, it drops very much from scattering photon-loss escape towards the Ly $\alpha \tau_{\mu}=1$ cloud surface which is very much further out in optical depth (Fig. 1). In addition, the large Wien $\mathrm{d} B / \mathrm{d} T$ 
non-linearity at $\operatorname{Ly} \alpha$ makes such surfaces very dark, resulting in underexposure unless bright areas are severely overexposed.

I suggest that these properties together explain the observed feature-less dark internetwork regions as in the lower-left corner of the Ly $\alpha$ image in Fig. 2.

Ly $\alpha$ does have adjacent-fibril opacity contrasts during the initial cooling phases while hydrogen recombines from full ionization if that was reached in the precursor PHE (as in the example of Pub II). I suggest that such contrasts together with high initial temperatures produce the observed short Ly $\alpha$ fibrils including "comet heads".

Obviously one desires $\mathrm{H} \alpha$-Ly $\alpha$ comparisons as in Fig. 2 but co-temporal, co-spatial and as a high-cadence time sequence, at the same resolution or yet better. Unfortunately, during the third VAULT Ly $\alpha$ flight on July 7, 2005 the shutter malfunctioned while the DOT was co-pointed during good seeing. The DOT mosaic in Fig. 2 was taken the next morning. The recent CLASP-1 rocket flight (Kano et al. 2016) yielded faster-cadence but lower-resolution Ly $\alpha$ images while the $\mathrm{H} \alpha$ observing at the Dunn Solar Telescope suffered from clouds. Regretfully, the $\mathrm{H} \alpha$-Ly $\alpha$ comparison in Fig. 2 remains the best there is.

In conclusion of this section: my conjecture to understand Fig. 2 is that Ly $\alpha$ shows PHEs as bright grains and the initial aftermaths of near-horizontally launched ones as short fibrils, whereas $\mathrm{H} \alpha$ shows their subsequent cooling tracks as long contrail fibrils with PSBE-defined contrasts. For such post-hot cooling features the Lyman and Ca II lines show the present, whereas their opacities in the Balmer lines and the Hiff continuum are defined by the hotter past.

\section{Discussion}

\subsection{Shock and post-shock visibilities}

Figure 1 suggests that the denser HION shocks become optically thick and therefore visible in $\mathrm{H} \alpha$. The subsequent PSBE lag suggests that cooling aftermath clouds are also visible in $\mathrm{H} \alpha$.

The actual existence of a HION-like shock-ridden domain in the internetwork areas of the solar atmosphere was established over two decades ago from $\mathrm{CaIIH} \mathrm{H}_{2}$ grains (e.g., Rutten \& Uitenbroek 1991; Carlsson \& Stein 1994; Carlsson \& Stein 1997); I then called it "clapotisphere" in a review (Rutten 1995). It is obvious in all internetwork areas in high-resolution $\mathrm{Ca}$ II $\mathrm{H}$ filtergram movies, e.g., those on my DOT movie page and on the Hinode quick-look movie pages and in ultraviolet continuum movies (e.g., Krijger et al. 2001), but it is very hard to detect in $\mathrm{H} \alpha$ (Rutten et al. 2008) in contrast to the $7000 \mathrm{~K}$ extinction expectation from Fig. 1. I attribute this surprising paucity of both shocks and cooling aftermaths to obscuration by overlying $\mathrm{H} \alpha$ contrail fibrils in which the gas has experienced higher temperatures than in shocks and retains PSBE non-transparency.

The similarities between $\mathrm{H} \alpha$ and the $\mathrm{H}$ Iff continua in Fig. 1 suggest comparable visibility and obscuration of clapotispheric shocks and their cooling aftermaths in ALMA diagnostics.

\subsection{Detection of contrail precursors}

My suggestion that most if not all long $\mathrm{H} \alpha$ fibrils are contrails requires ubiquitous precursor PHEs that have not yet been identified, but may be similar to the small fast heating events of De Pontieu et al. (2011), Tian et al. (2011), Scullion et al. (2015), Shetye et al. (2016).
The hot precursor of the contrail fibril of Pub II was visible as an extending bright streak in $1400 \AA$ slitjaw images from IRIS, in yet hotter diagnostics from SDO/AIA, and in the far blue wing of $\mathrm{H} \alpha$ as a dark streak due to large blueshift and thermal core broadening. It may have gotten its joint visibility in these diverse diagnostics by being relative opaque, large, and slow. Smaller events may lose visibility from smaller optical thickness or insufficient angular resolution.

The question so arises which diagnostics suit best to spot smaller, less opaque, possibly faster heating events than RBEs, RREs, and the contrail producer of Pub II. The SB curves in Fig. 1 suggest much higher extinction coefficient for hot PSBE precursors in $\operatorname{Ly} \alpha$ and the mm wavelengths than for $\mathrm{H} \alpha$, also higher than in other chromospheric lines (Fig. 5 of Pub I). This suggests that Ly $\alpha$ and $\mathrm{mm}$ wavelengths are the best to find them, with largest opacity and emissivity in Ly $\alpha$ where hydrogen remains partially neutral and largest opacity and emissivity at $\mathrm{mm}$ wavelengths at full hydrogen ionization. In the absence of a fastcadence high-resolution Ly $\alpha$ space mission ALMA is the most promising facility if it reaches sufficient angular resolution.

Intrinsic solar-atmosphere scattering blurs such precursors to larger apparent extent in Ly $\alpha$ images, but not at mm wavelengths so that for ALMA the required angular resolution to detect PHEs as small bright blobs or jets is higher than in Ly $\alpha$. ALMA will show them in the detail permitted by the array resolution. At only partial hydrogen ionization $\operatorname{Ly} \alpha$ scattering from the precursor PHE into cooler surrounding gas will produce dark opaque aureoles around such bright kernels in ALMA images.

\subsection{Fibrils with ALMA}

Hotter precursors than HION shocks will leave larger PSBE opacities since higher temperature implies larger $\varepsilon$ in Ly $\alpha$. The precursor in the contrail of Pub II must have reached temperatures above $10000 \mathrm{~K}$ since it was visible in UV IRIS and EUV AIA images. Near this temperature the SB H $\alpha$ curves reach their peak while the H I ff curves reach yet higher saturation levels. If the postulated precursors become this hot or even hotter, then the non-E-retarded contrail opacity in the post-event cooling phase will be much larger for the mm continua than for $\mathrm{H} \alpha$. I therefore expect $\mathrm{mm}$ contrail fibrils to be yet more opaque than the $\mathrm{H} \alpha$ ones, constituting a yet denser canopies.

\subsection{Fibrils with Bifrost}

The most elaborate studies of $\mathrm{H} \alpha$ fibril formation in numerical simulations of the solar atmosphere are those by Leenaarts et al. (2012, 2015), using a snapshot of a 3D non-E MHD simulation with the Bifrost code (Gudiksen et al. 2011) that was later made public by Carlsson et al. (2016). The same snapshot was used in predictions for ALMA by Loukitcheva et al. (2015).

This snapshot shows some $\mathrm{H} \alpha$ fibrils on the condition that the spectral synthesis is done with $3 \mathrm{D}$ radiative transfer so that the larger-contrast granulation signature imposed by thermal photon creation in the deep photosphere is erased by scattering within the overlying chromosphere (Fig. 7 of Leenaarts et al. 2012). This implies that the Bifrost fibrils are less opaque than actual solar fibrils which do not let even very bright Ellerman bombs shine through at line center. Also, the synthetic $\mathrm{H} \alpha$ image shows far fewer $\mathrm{H} \alpha$ fibrils than areas with a similar amount of magnetism would show in actual observations. They mostly connect opposite-polarity network patches; none jut out far over adjacent internetwork. 
I suspect that these differences are due to various deficiencies in the Bifrost studies. First, although the MHD simulation accounted for non-equilibrium hydrogen ionization, the $\mathrm{H} \alpha$ synthesis did not by applying statistical-equilibrium NLTE on the single snapshot so that the $\mathrm{H} \alpha$ opacities had no posthot boosting. The relatively few Bifrost fibrils gained $\mathrm{H} \alpha$ and ALMA contrasts only from density differences, not from memorial opacities. There was also no opacity spreading from Ly $\alpha$ scattering since the net radiative rates of the Lyman transitions were assumed zero for tractability as in the HION atmosphere of Leenaarts et al. (2007).

Second, the non-equilibrium increase of the electron density that resulted from retarded hydrogen recombination in the simulation and was retained in the snapshot analysis tends to force hydrogen back to the H I ground level, just as in SB equilibrium, giving smaller $\mathrm{H} \alpha$ extinction than without such non-E electron density increase.

Finally, while Bifrost simulations do an outstanding job in emulating solar granulation, acoustic waves and shocks, network-like field concentrations, dynamic fibrils, and more of the rich zoo of solar-atmosphere phenomena, they have not yet produced spicules-II or equivalent RBEs and RREs. It is not for me to elucidate why Bifrost fails to make these, but I do speculate that Bifrost also does not make the smaller and hotter PHEs that I deem required to explain ubiquitous $\mathrm{H} \alpha$ fibrils.

About the same few fibrils appeared in the longerwavelength synthetic ALMA-prediction images in Fig. 4 of Loukitcheva et al. (2015). These resulted from evaluation of the integral LTE transfer equation in terms of brightness temperature using H I ff opacities given by the non-E electron and proton densities in the simulation. However, long fibrils that extend away from the network areas are also missing in these synthetic images.

Since the actual Sun is largely covered by $\mathrm{H} \alpha$ fibrils and Fig. 1 predicts that these will be as opaque at $\mathrm{mm}$ wavelengths in the case of instantaneous opacities and much more opaque in the case of PSBE memories, I wouldn't trust any fibril-lacking simulation to suggest what ALMA will observe in fibrilar areas.

\subsection{Fibril widths}

Resonance scattering of the intense Ly $\alpha$ radiation from recombining gas along PHE precursor tracks boosts $\mathrm{H} \alpha$ and $\mathrm{H}$ I freefree extinction over a few hundred $\mathrm{km}$ in cooler gas around the tracks (Fig. 3 of Pub I, display 26: aureole boosting). Contrail fibrils therefore show widths of this extent in $\mathrm{H} \alpha$ even when the actual precursors are much smaller and harder to detect - just as aircraft jet engines are smaller on the sky and harder to observe than the contrails they produce. ALMA images will show them opaque over similar or yet larger width, but render the actual cross-section temperature profile as brightness.

\subsection{Fibril temperatures}

I base a rough estimate of contrail fibril temperatures on a comparison of the CaII $8542 \AA$ and $\mathrm{H} \alpha$ SB extinction curves in Fig. 1 with the observational Ca II $8542 \AA-\mathrm{H} \alpha$ comparison for a quiet-Sun scene in Cauzzi et al. (2009). It showed that fibrils appear similarly in both lines near network, but in their jutting out across internetwork they quickly become transparent in Ca II $8542 \AA$ whereas they extend further out in $\mathrm{H} \alpha$. As a result, the brightness-brightness scatter plot in the first column of Fig. 6 of that article shows only correlation for the brightest network samplings. However, the same figure shows high correlations between $\mathrm{H} \alpha$ core width, Ca II $8542 \AA$ core width, and Ca II $8542 \AA$ Dopplershift-following line-minimum intensity were these quantities are not small. Cauzzi et al. (2009) attributed these good correspondences to joint temperature sampling. For the $\mathrm{H} \alpha$ core width this was expected because its thermal broadening is relatively large due to the small atomic mass of hydrogen; for Ca II $8542 \AA$ core width and intensity it was not surprising since both are temperature-sensitive. The maps of these quantities in Figs. 3 and 4 in that article then suggest lower fibril temperatures further away from network.

The H $\alpha$ and Ca II $8542 \AA$ curves in Fig. 1 cross at 6000$7000 \mathrm{~K}$, implying that where chromospheric fibrils appear similar in these lines their temperature is about this value. This so becomes my prediction for temperatures to be measured with ALMA in the initial parts of long fibrils. At these high temperatures non-E retardation is small. Adjacent-fibril contrast in line-core images comes from different densities, different temperatures in the case of Ca II $8542 \AA$, and especially from different Dopplershifts with larger sensitivity for the relatively narrow core of Ca II $8542 \AA$.

Since further away from activity the actual fibrils become cooler, the Ca II $8542 \AA$ and H $\alpha$ opacities along them get smaller in the case of instantaneous SB population following the leftward declines in Fig. 1. This decline is much steeper for $\mathrm{H} \alpha$ due to its large Boltzmann sensitivity, so that in quiescent conditions $\mathrm{H} \alpha$ fibrils should become transparent and invisible well before Ca II 8542 A fibrils and therefore should appear shorter - instead of being longer as observed.

My remedy for this incongruity is again to postulate that hotter events have passed previously, making the $\mathrm{H} \alpha$ extinction coefficient initially much higher (up the $\mathrm{H} \alpha$ slopes towards the peaks in Fig. 1) and that the post-event cooling gas retains such high $\mathrm{H} \alpha$ extinction for minutes while the Ca II $8542 \AA$ extinction adjusts near-instantaneously to the decreasing temperature.

The observed transparency of outer fibrils in Ca II $8542 \AA$ then suggests actual fibril temperatures around $5000 \mathrm{~K}$ or less away from network, with long retardation and PSBE opacities in $\mathrm{H} \alpha$ and at mm wavelengths.

The brightness temperatures in ALMA data should directly correspond to these temperature predictions. In $\mathrm{H} \alpha$ the corresponding brightness-temperature range is much smaller, covering only $4000-4200 \mathrm{~K}$ in Fig. 6 of Cauzzi et al. (2009), because it is set by scattering. Nevertheless, contrail fibrils will have good dark-dark correspondence in byte-scaled ALMA-H $\alpha$ brightness comparisons because low fibril temperature translates directly into low mm brightness but also indirectly into low $\mathrm{H} \alpha$ brightness via large PSBE opacity.

The arguments above for the Ca II $8542 \AA-\mathrm{H} \alpha$ incongruity were earlier used in Rutten et al. (2008) to explain the lack of fibril-canopy signature at the center of $\mathrm{Ca}$ II $\mathrm{H}$ in the venerable spectrogram sequence of Lites et al. (1993). Although these spectrograms became famous through the Radyn internetwork oscillation emulation by Carlsson \& Stein (1997), the original analysis concentrated on long-period network oscillations at $\mathrm{Ca}$ II $\mathrm{H}$ center that in hindsight may have been a signature of repetitive PHE launching seen as accelerating Dopplergram branches jutting out from network in Fig. 2 of Rutten et al. (2008), possibly producing the thin Ca II K canopy fibrils reported by Pietarila et al. (2009) that seem to correspond to thin straws near the limb (Fig. 1 of Rutten 2006) and off-limb spicules-II (De Pontieu et al. 2007b). 
Finally, it is impossible to quantify the temperature of the postulated PHE precursors because they have not yet been identified. However, the visibility of the contrail precursor of Pub II in UV IRIS and EUV AIA images suggests that such events may become significantly hotter than $10000 \mathrm{~K}$.

\section{Conclusion}

The demonstration in Fig. 4 of Wedemeyer (2016) that ALMA "can serve as a linear thermometer for the chromospheric plasma" is obviously correct, but I suggest that scenes as the simulation-predicted clapotisphere depicted in that figure will hardly be detected by ALMA, just as they are hard to find in $\mathrm{H} \alpha$.

In summary, Fig. 1 shows that if $\mathrm{H} \alpha$ fibrils are instantaneous $7000 \mathrm{~K}$ features (as is the ALC7 chromosphere) then their opacities will be similar to those in $\mathrm{H} \alpha$ at $0.35 \mathrm{~mm}$, larger at $1.3 \mathrm{~mm}$, and much larger at $3.0 \mathrm{~mm}$. If instead most long $\mathrm{H} \alpha$ fibrils represent retarded-opacity features after heating events as the one in Pub II, i.e., are contrails as I suggest from the striking scene difference in Fig. 2, the paucity of $\mathrm{H} \alpha$ shock scenes and the fibril incongruity with Ca II $8542 \AA$, then their opacities are very much larger at all ALMA wavelengths.

My prediction is that in ALMA images most of the solar surface will be covered by opaque $\mathrm{H} \alpha$-like fibrils. I think it naive to ignore the observed $\mathrm{H} \alpha$ truth when basing predictions for ALMA on solar-atmosphere simulations that lack the ubiquitous fibrilar $\mathrm{H} \alpha$ canopies.

However, on a more positive note, the fibrilar chromosphere and especially the proposed precursor heating events represent a much more interesting and promising research topic than internetwork shocks which are well understood since Carlsson \& Stein (1994) and do not play an important role in solar atmosphere heating (Carlsson \& Stein 1995).

Because solar physics is a field of too scarce predictions I summarize this study with a dozen specific ones:

1. although probably the Sun will be less active when ALMA starts solar observing, I predict that most of the solar surface will be covered by long opaque fibrils in ALMA images;

2. more precisely, I predict that at the ALMA wavelengths the general appearance of the Sun will be similar to $\mathrm{H} \alpha$ images with good dark-dark correspondence but with larger fibril opaquenesses at $\mathrm{mm}$ wavelengths that increase with wavelength and with less lateral fibril contrast due to insensitivity to Dopplershifts;

3. yet more precisely, I predict that the actual fibril temperatures fall into three categories: above $10000 \mathrm{~K}$ in small heating events propagating outward from activity, around $7000 \mathrm{~K}$ in the initial parts of resulting fibrils, and cooling down to $5000 \mathrm{~K}$ and less along subsequent long contrails emanating far across internetwork;

4. while ALMA can easily quantify the second and third temperature categories, the first consists of difficult, hard-tocatch features. However, even while small and fast, such events have opacities at $\mathrm{mm}$ wavelengths that are much larger than in $\mathrm{H} \alpha$ (at high temperature even larger than in $\operatorname{Ly} \alpha$ ), making them best detectable in fast-cadence image sequences from ALMA if these reach sufficient angular resolution. I optimistically predict that ALMA will see them. Measuring their temperature, energy release, and contribution to atmospheric heating is then an exciting ALMA quest: something new on the Sun - more attractive than detailing well-known non-heating acoustic shocks;

5. if ALMA indeed detects such precursor heating events then I predict that these initially possess darker opaque aureoles from Ly $\alpha$ scattering and that such sunny-side-up morphology vanishes from complete hydrogen ionization within the precursor at ALMA-measured temperatures below $15000 \mathrm{~K}$, closer to the SB than the CE limit;

6. I predict that even when the hot precursors are very small they produce contrail widths of order $0.5 \operatorname{arcsec}$ through Ly $\alpha$ scattering;

7. I predict that the precursor events are better field mappers from line tying while hydrogen gets ionized than subsequent less tied and wider contrail fibrils;

8. I predict that ALMA will only sample internetwork shocks in rare, utterly quiet areas free of fibrilar obscuration and there will also detect subsequent cooling clouds with temperatures dropping below $4000 \mathrm{~K}$ conform the COmosphere of Ayres \& Rabin (1996);

9. I predict that ALMA will not observe any Ellerman bomb. They will be as obscured by fibril canopies as at the center of $\mathrm{H} \alpha$;

10. more positively, I predict that ALMA will observe more forceful reconnection events that break through or occur above the fibril canopies and contribute coronal heating, in particular the flaring active-region fibrils described in Vissers et al. (2015) and Pub I. So far they are best seen in ultraviolet continua but they can also appear as IRIS bombs in ultraviolet lines (Peter et al. 2014; Vissers et al. 2015). It is of great interest to track their temperature;

11. I predict that spicules-II will be much more opaque in offlimb imaging with ALMA than in $\mathrm{H} \alpha$ and $\mathrm{Ca}$ II $\mathrm{H}$;

12. similarly, I predict that wherever coronal rain is opaque in $\mathrm{H} \alpha$ it will be much more opaque at mm wavelengths.

Hopefully these predictions will soon be verified with actual ALMA observations. I look forward to be proven right or wrong.

I end this study with a speculation. Quiescent filaments, in particular threads in their barbs and legs, may likewise obtain their extraordinary $\mathrm{H} \alpha$ visibility from post-hot opacity produced by frequent small heating events. They will then have yet larger opacities and show up thicker in ALMA images, at lower temperatures than suggested by statistical-equilibrium $\mathrm{H} \alpha$ modeling.

Acknowledgements. I am much indebted to S. Toriumi of the National Astronomical Observatory of Japan for inviting me for an extended stay and being an excellent and efficient host. This analysis resulted from illuminating and inspiring discussions there with R. Ishikawa, M. Kubo, M. Shimojo and T.J. Okamoto. I thank the referee for suggesting many presentation improvements. CHIANTI is a project of George Mason University, the University of Michigan and the University of Cambridge. I made much use of the SolarSoft and ADS libraries. My LaTeX macro to make in-text citations link to ADS was improved by EDP Sciences; the trick to link to specific pages came from E. Henneken at ADS. The EDP production of A\&A also conserves my definition popups for acronyms.

\section{References}

Avrett, E. H. 1965, SAO Spec. Rep., 174, 101

Avrett, E. H., \& Loeser, R. 2008, ApJS, 175, 229

Ayres, T. R., \& Rabin, D. 1996, ApJ, 460, 1042

Carlsson, M., \& Stein, R. F. 1994, in Chromospheric Dynamics, ed. M. Carlsson, 47

Carlsson, M., \& Stein, R. F. 1995, ApJ, 440, L29 
Carlsson, M., \& Stein, R. F. 1997, ApJ, 481, 500

Carlsson, M., \& Stein, R. F. 2002, ApJ, 572, 626

Carlsson, M., Hansteen, V. H., Gudiksen, B. V., Leenaarts, J., \& De Pontieu, B 2016, A\&A, 585, A4

Cauzzi, G., Reardon, K., Rutten, R. J., Tritschler, A., \& Uitenbroek, H. 2009, A\&A, 503, 577

De Pontieu, B., Hansteen, V. H., Rouppe van der Voort, L., van Noort, M., \& Carlsson, M. 2007a, ApJ, 655, 624

De Pontieu, B., McIntosh, S., Hansteen, V. H., et al. 2007b, PASJ, 59, S655

De Pontieu, B., McIntosh, S. W., Carlsson, M., et al. 2007c, Science, 318, 1574

De Pontieu, B., McIntosh, S. W., Carlsson, M., et al. 2011, Science, 331, 55

Dere, K. P., Landi, E., Mason, H. E., Monsignori Fossi, B. C., \& Young, P. R. 1997, A\&AS, 125, 149

Gudiksen, B. V., Carlsson, M., Hansteen, V. H., et al. 2011, A\&A, 531, A154

Hansteen, V. H., De Pontieu, B., Rouppe van der Voort, L., van Noort, M., \& Carlsson, M. 2006, ApJ, 647, L73

Jefferies, J. T., \& Thomas, R. N. 1959, ApJ, 129, 401

Joselyn, J., Munro, R. H., \& Holzer, T. E. 1979, Sol. Phys., 64, 57

Judge, P., \& Centeno, R. 2008, ApJ, 687, 1388

Kano, R., Ishikawa, R., Winebarger, A. R., et al. 2016, in AAS/Solar Physics Division Meeting, 47,\#101.07

Klein, R. I., Kalkofen, W., \& Stein, R. F. 1976, ApJ, 205, 499

Kneer, F. 1980, A\&A, 87, 229

Kneer, F., \& Nakagawa, Y. 1976, A\&A, 47, 65

Koza, J., Rutten, R. J., \& Vourlidas, A. 2009, A\&A, 499, 917

Krijger, J. M., Rutten, R. J., Lites, B. W., et al. 2001, A\&A, 379, 1052

Landi, E., Young, P. R., Dere, K. P., Del Zanna, G., \& Mason, H. E. 2013, ApJ, 763,86

Langangen, Ø., De Pontieu, B., Carlsson, M., et al. 2008, ApJ, 679, L167

Leenaarts, J., \& Wedemeyer-Böhm, S. 2006, in Solar MHD Theory and Observations: A High Spatial Resolution Perspective, eds. J. Leibacher, R. F. Stein, \& H. Uitenbroek, ASP Conf. Ser., 354, 306

Leenaarts, J., Carlsson, M., Hansteen, V., \& Rutten, R. J. 2007, A\&A, 473, 625

Leenaarts, J., Carlsson, M., \& Rouppe van der Voort, L. 2012, ApJ, 749, 136

Leenaarts, J., Carlsson, M., \& Rouppe van der Voort, L. 2015, ApJ, 802, 136

Lites, B. W., Rutten, R. J., \& Kalkofen, W. 1993, ApJ, 414, 345

Loukitcheva, M., Solanki, S. K., Carlsson, M., \& White, S. M. 2015, A\&A, 575, A15

Mihalas, D. 1967, in Astrophysics, eds. B. Alder, S. Fernbach, \& M. Rotenberg (New York: Academic Press), Meth. Comp. Phys., 7, 1

Mihalas, D. 1970, Stellar atmospheres (San Francisco: Freeman)

Patsourakos, S., Gouttebroze, P., \& Vourlidas, A. 2007, ApJ, 664, 1214

Pereira, T. M. D., De Pontieu, B., \& Carlsson, M. 2012, ApJ, 759, 18

Peter, H., Tian, H., Curdt, W., et al. 2014, Science, 346, 1255726

Pietarila, A., Hirzberger, J., Zakharov, V., \& Solanki, S. K. 2009, A\&A, 502, 647

Poletto, G. 1979, Sol. Phys., 61, 389

Rouppe van der Voort, L. H. M., De Pontieu, B., Hansteen, V. H., Carlsson, M., \& van Noort, M. 2007, ApJ, 660, L169
Rouppe van der Voort, L., Leenaarts, J., De Pontieu, B., Carlsson, M., \& Vissers, G. 2009, ApJ, 705, 272

Rouppe van der Voort, L., \& de la Cruz Rodríguez, J. 2013, ApJ, 776, 56

Rutten, R. J. 1995, in Helioseismology, ESA SP, 376, 151

Rutten, R. J. 2003, Radiative Transfer in Stellar Atmospheres, Utrecht University Lecture Notes

Rutten, R. J. 2006, in Solar MHD Theory and Observations: A High Spatial Resolution Perspective, eds. J. Leibacher, R. F. Stein, \& H. Uitenbroek, ASP Conf. Ser. 354, 276

Rutten, R. J. 2007, in The Physics of Chromospheric Plasmas, eds. P. Heinzel, I. Dorotovič, \& R. J. Rutten, Heinzel, ASP Conf. Ser., 368

Rutten, R. J. 2016, A\&A, 590, A124

Rutten, R. J., \& Carlsson, M. 1994, in Infrared Solar Physics, eds. D. M. Rabin, J. T. Jefferies, \& C. Lindsey, IAU Symp., 154, 309

Rutten, R. J., \& Uitenbroek, H. 1991, Sol. Phys., 134, 15

Rutten, R. J., \& Uitenbroek, H. 2012, A\&A, 540, A86

Rutten, R. J., Hammerschlag, R. H., Bettonvil, F. C. M., Sütterlin, P., \& De Wijn, A. G. 2004, A\&A, 413, 1183

Rutten, R. J., van Veelen, B., \& Sütterlin, P. 2008, Sol. Phys., 251, 533

Rutten, R. J., Vissers, G. J. M., Rouppe van der Voort, L. H. M., Sütterlin, P., \& Vitas, N. 2013, J. Phys. Conf. Ser., 440, 012007

Rutten, R. J., \& Rouppe van der Voort, L. H. M. 2017, A\&A, 597, A138 (Pub II)

Rybicki, G. B., \& Lightman, A. P. 1986, Radiative Processes in Astrophysics (Wiley-VCH)

Sánchez Almeida, J. 1992, Sol. Phys., 137, 1

Sánchez Almeida, J. 1997, ApJ, 491, 993

Scullion, E., Engvold, O., Lin, Y., \& Rouppe van der Voort, L. 2015, ApJ, 814, 123

Sekse, D. H., Rouppe van der Voort, L., \& De Pontieu, B. 2012, ApJ, 752, 108

Sekse, D. H., Rouppe van der Voort, L., De Pontieu, B., \& Scullion, E. 2013, ApJ, 769, 44

Shetye, J., Doyle, J. G., Scullion, E., et al. 2016, A\&A, 589, A3

Thomas, R. N. 1957, ApJ, 125, 260

Tian, H., McIntosh, S. W., De Pontieu, B., et al. 2011, ApJ, 738, 18

Uitenbroek, H. 2001, ApJ, 557, 389

Vernazza, J. E., Avrett, E. H., \& Loeser, R. 1981, ApJS, 45, 635

Vissers, G. J. M., Rouppe van der Voort, L. H. M., Rutten, R. J., Carlsson, M., \& De Pontieu, B. 2015, ApJ, 812, 11

Vourlidas, A., Sánchez Andrade-Nuño, B., Landi, E., et al. 2010, Sol. Phys., 261, 53

Wedemeyer, S. 2016, The Messenger, 163, 15

Wedemeyer, S., Bastian, T., Brajša, R., et al. 2016a, Space Sci. Rev., 200, 1

Wedemeyer, S., Fleck, B., Battaglia, M., et al. 2016b, ArXiv e-prints [arXiv: 1601.00587]

Wedemeyer-Böhm, S., Ludwig, H. G., Steffen, M., Leenaarts, J., \& Freytag, B. 2007, A\&A, 471, 977

Wittmann, A. 1974, Sol. Phys., 35, 11 


\section{SOLAR SPECTRUM FORMATION: EXAMPLES}

Robert J. Rutten

http://www.staff.science.uu.nl/ rutte101

The clickers below link to specific pages in this pdf file. It is a snapshot selection from my example displays made on October 42016 by retaining only those that concern the ALC7 model atmosphere and non-equilibrium $\mathrm{H} \alpha$ formation. Many are hyperlinked in my publication "Solar Ho features with hot onsets III. Long fibrils in Ly $\alpha$ and with ALMA" accepted for Astronomy \& Astrophysics on that date (DOI http: / / dx . doi . org/ 10 . 1051/0004-6361/201629238).

My current collection of lecture displays resides at:

http://www.staff.science.uu.nl/ rutte101/Lectures.html

ALC7 model: model hydrogen versus FCHHT-B strong lines

explanation of line plots: pops plot BSJ plot profile plot

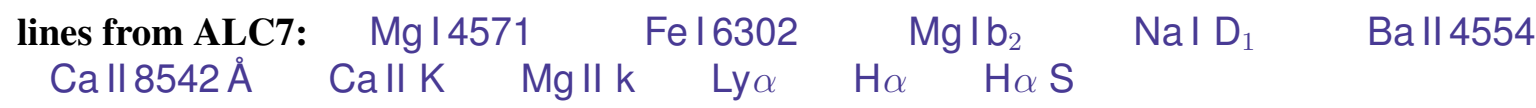

dynamic chromosphere: non-E H $\alpha$ aureole boosting $\quad \mathrm{H} \alpha$ extinction

hidden clickers: title $=$ previous page $\quad$ top-left $=$ start $\quad$ top-right $=$ thumbnails 


\section{ALC7 ATMOSPHERE}

Avrett \& Loeser 2008ApJS..175..229A

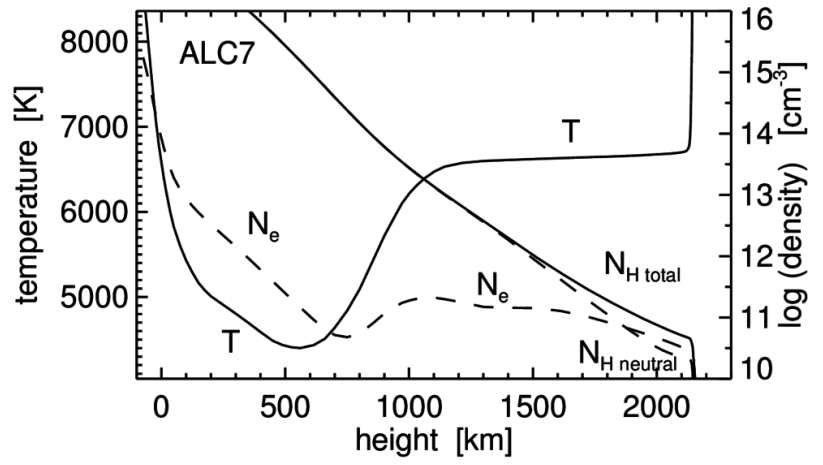

- unrealistic plane-parallel static computational star with solar-like average spectrum - exemplary in obeying all equations in my RT courses: understandable line formation

- best-fit temperature: near-RE in photosphere, shock-dominated in chromosphere - slope in upper photosphere depends on NLTE ultraviolet line haze

- total hydrogen density: exponential decay

- turbulent presssure added to gain scale height and chromospheric extent

- low electron density in photosphere and temperature minimum - from ionization of donor-elements $\mathrm{Si}, \mathrm{Fe}, \mathrm{Mg}, \mathrm{Al}$ with $10^{-4}$ relative abundance

- increasing hydrogen ionization across chromosphere - electron density reaches proton density at its top

- near-isothermal near-constant- $N_{\mathrm{e}}$ chromosphere - mimics Avrett's (1965) isothermal constant- $\varepsilon$ two-level-atom scattering atmosphere 


\section{CRD RESONANT SCATTERING IN AN ISOTHERMAL ATMOSPHERE}

RTSA figure 4.12; from Avrett 1965SAOSR.174..101A
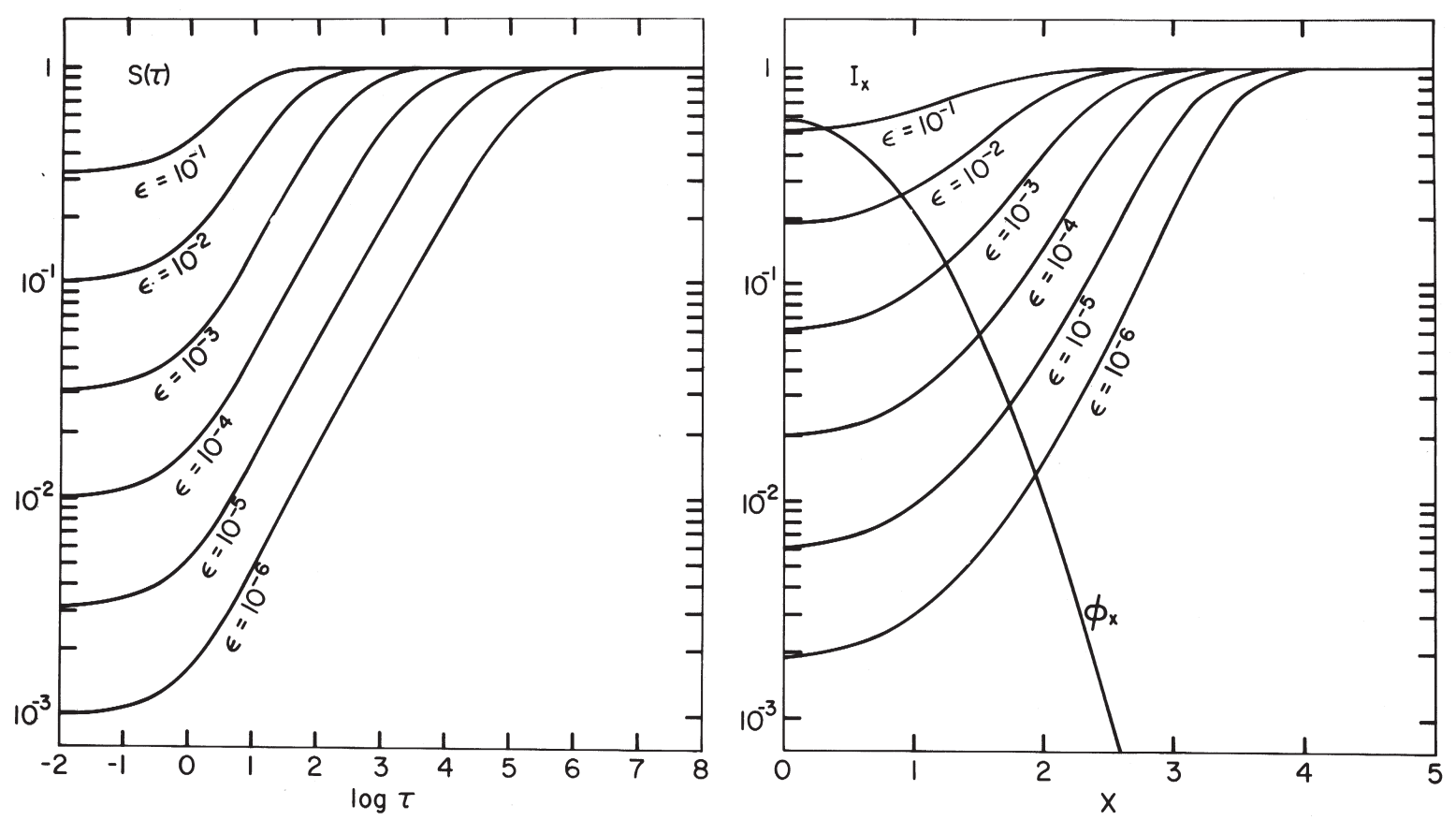

- left: $S / B$ in a plane-parallel isothermal atmosphere with constant $\varepsilon$ for complete redistribution. The curves illustrate the $\sqrt{\varepsilon}$ law and thermalization at $\Lambda \approx 1 / \varepsilon$.

- right: corresponding emergent line profiles and Gaussian extinction profile shape $\phi$ (only the righthand halves; $x=\Delta \lambda / \Delta \lambda_{\mathrm{D}}$ ) 


\section{HYDROGEN LINES IN THE ALC7 ATMOSPHERE}
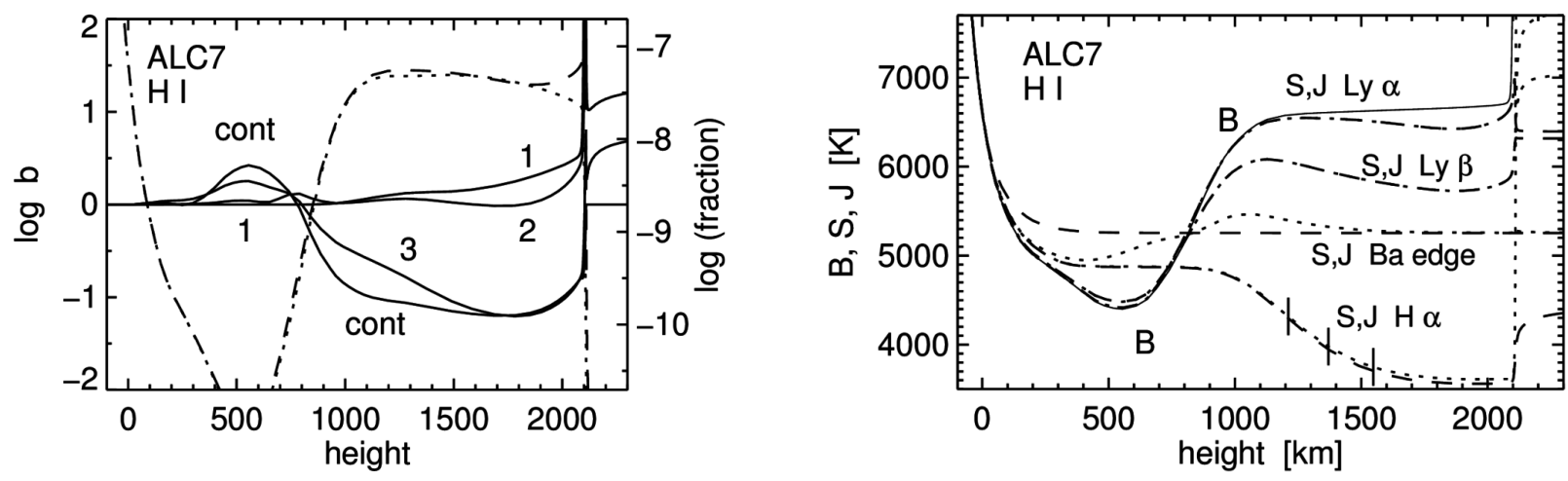

$\mathrm{H} \alpha$ : chromosphere is back-scattering attenuator for radiation from deep photosphere; outward $S$ decline as in isothermal constant- $\varepsilon$ two-level-atom atmosphere

Ly $\alpha$ : tremendous scattering with $S_{\mathrm{Ly} \alpha} \approx J_{\mathrm{Ly} \alpha}$ but local thermalization with $J_{\mathrm{Ly} \alpha} \approx B_{\mathrm{Ly} \alpha}$ from short photon mean free paths ( $S$ dotted, $J$ dashed; dot-dashed $=$ identity)

Ly $\beta$ : scattering as $\mathrm{Ly} \alpha$, shares photon losses in $\mathrm{H} \alpha$ ( $\mathrm{H} \alpha$ ticks $\tau=3,1,0.3$ ) (same $S / B \approx b_{3} / b_{l}$ since $b_{2} \approx b_{1}$ but offsets differ in temperature representation)

$n=1$ : Saha-Boltzmann $b_{1} \approx 1$ population because hydrogen is neutral (except in transition region at right)

$n=2$ : Saha-Boltzmann $b_{2} \approx 1$ population from Ly $\alpha$ thermalization (dotted fraction curve $=n_{2}^{\mathrm{LTE}} / N_{\mathrm{Htot}} \approx$ dashed curve $=$ actual $n_{2} / N_{\mathrm{Htot}}$ )

ionization: $b_{\text {cont }} / b_{2}$ defined by SE balancing of $B\left(T_{\text {rad }}^{\text {Bacont }}\right) / B\left(T_{\mathrm{e}}\right)$ ionization driving and recombination driving by Balmer and higher line photon losses. The HI top $(n \geq 2)$ represents a $3.4 \mathrm{eV}$ alkali atom with ground-state population set by Ly $\alpha$. 
5 EXPLAIN EVERYTHING - INCLUDING SIMILARITIES AND DIFFERENCES
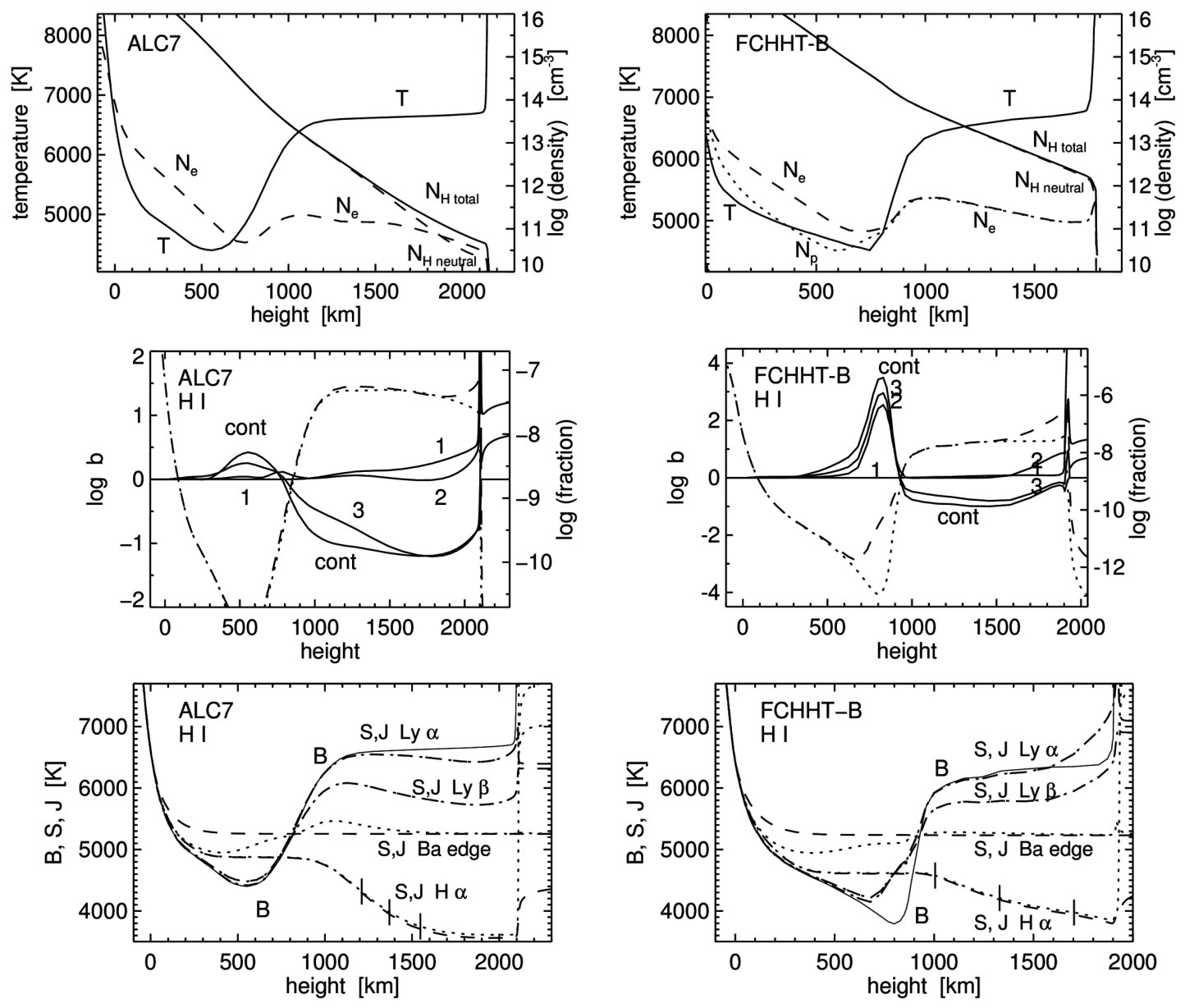


\section{STRONG LINES IN ALC7}

Avrett \& Loeser 2008ApJS..175..229A

Rutten 2016A\&A...590A.124R
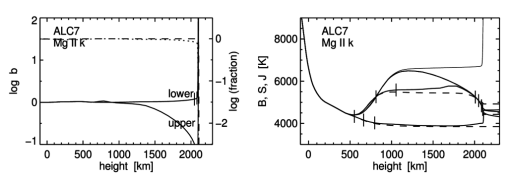

- $M g / l k$

- extinction LTE, source function 2-level scattering

- high peaks, low PRD dips, low wings
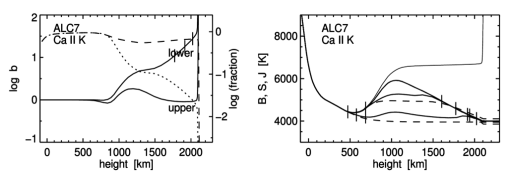

- Call K

- lower abundance and ionization, underionization

- small peaks and PRD dips

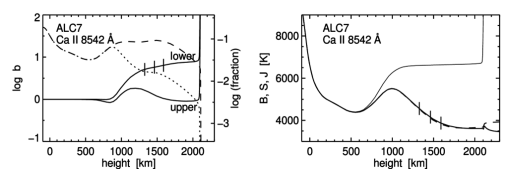

- Ca ll 8542

- as Call K with Boltzmann lowering and sensitivity

- similar source function sampling as $\mathrm{H} \alpha$

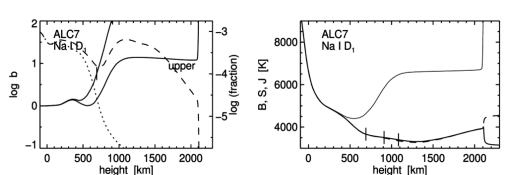

- $\mathrm{Na} / D_{1}$

- photospheric scattering, suction and underionization

- no sensitivity to temperature rise

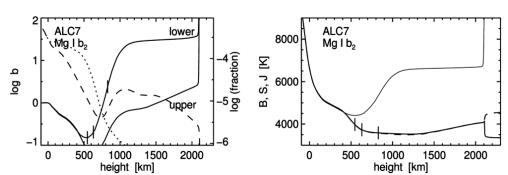

- $M g / b_{2}$

- as Nal $D_{1}$ but photospheric overionization

- no sensitivity to temperature rise

- $H_{\alpha}$

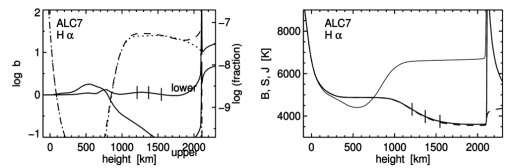

- chromospheric scattering of photospheric photons

- chromospheric extinction LTE from Ly $\alpha$ box-up 
7 LINES FROM THE ALC7 ATMOSPHERE: POPULATIONS PLOT (NaI D $\mathrm{D}_{1}$
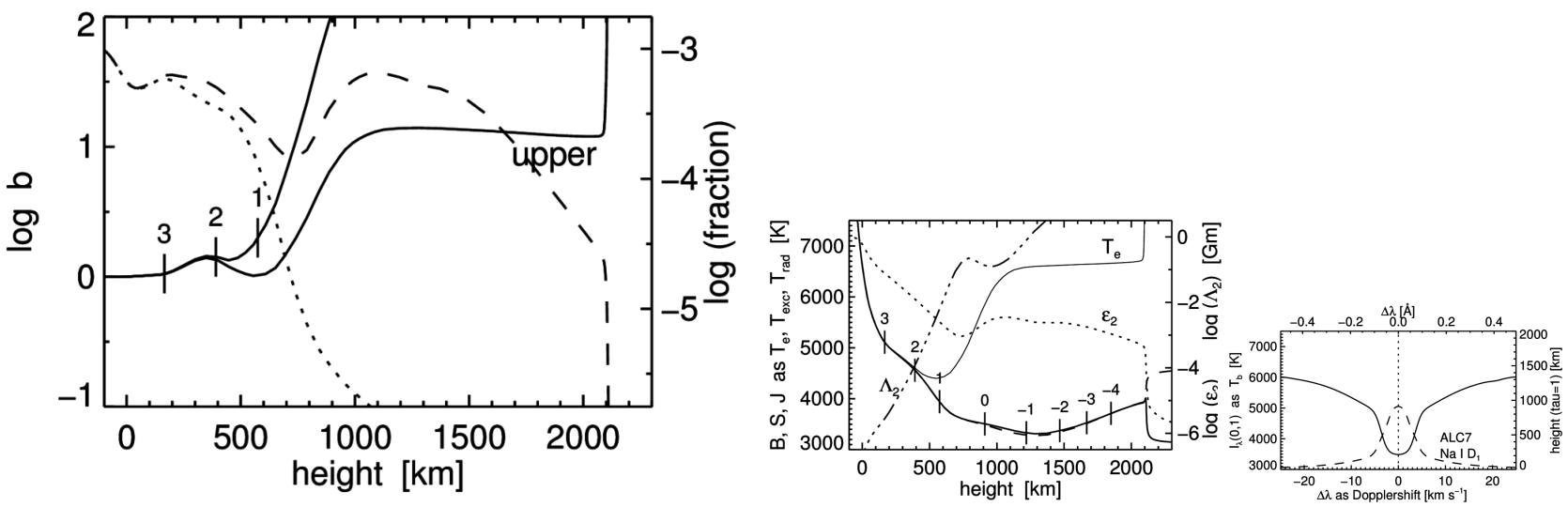

$$
b_{i} \equiv n_{i} / n_{i}^{\mathrm{LTE}} \quad \alpha^{l} \approx b_{l} \alpha^{\mathrm{LTE}} \quad S^{l} \approx\left(b_{u} / b_{l}\right) B \quad \varepsilon \approx \varepsilon_{2}=\alpha^{\mathrm{a}} /\left(\alpha^{\mathrm{s}}+\alpha^{\mathrm{a}}\right) \quad S^{l} \approx\left(1-\varepsilon_{2}\right) \bar{J}+\varepsilon_{2} B
$$

- solid: population departure coefficients for $\mathrm{NaI} \mathrm{D}_{1}$. Unity in deep photosphere from large collision frequency at high density, with $\varepsilon \approx 1$ ( $B S J$ plot). Increasing $b_{u}<b_{l}$ divergence $=$ $S^{l}<B$ divergence ( $B S J$ plot) from $\sqrt{\varepsilon}$-law resonance scattering. Small initial hump in upper photosphere from photon suction (replenishment from ion reservoir) by scattering-out $\mathrm{NaI} D$ photons. Steep $b_{l}$ rise above $700 \mathrm{~km}$ from ultraviolet underionization $(1-c$ edge at $2412 \AA$, typical for minority neutrals). The $\log \tau$ ticks on the $b_{l}$ curve are for line center.

- dotted: fractional population $n_{l}^{\mathrm{LTE}} / N_{\text {elem }}$ per Saha-Boltzmann. Scale at right. $\mathrm{Nal}$ is a minority species. Initial decrease from increasing ionization at decreasing $N_{\mathrm{e}}$, slight hump from less ionization at lower temperature, steep decline at increasing $T$ and decreasing $N_{\mathrm{e}}$ (Saha).

- dashed: fractional population $n_{l} / N_{\text {elem }}$ in NLTE. Line-center optical depth $\tau_{\lambda}=-\int\left(\alpha^{l}+\alpha^{c}\right) \mathrm{d} h$ has $\alpha^{l}>>\alpha^{c}$ and $\alpha_{\lambda}^{l} \sim n_{l}=\left(n_{l} / N_{\text {elem }}\right) A_{\text {elem }} N_{\text {Htot }}$. Divergence from LTE curve corresponds to departure of $b_{l}$ from unity. The steep $b_{l}$ increase compensates the steep $n_{l}^{\mathrm{LTE}}$ decrease. 


\section{LINES FROM THE ALC7 ATMOSPHERE: B S J PLOT (Na I D 1 )}

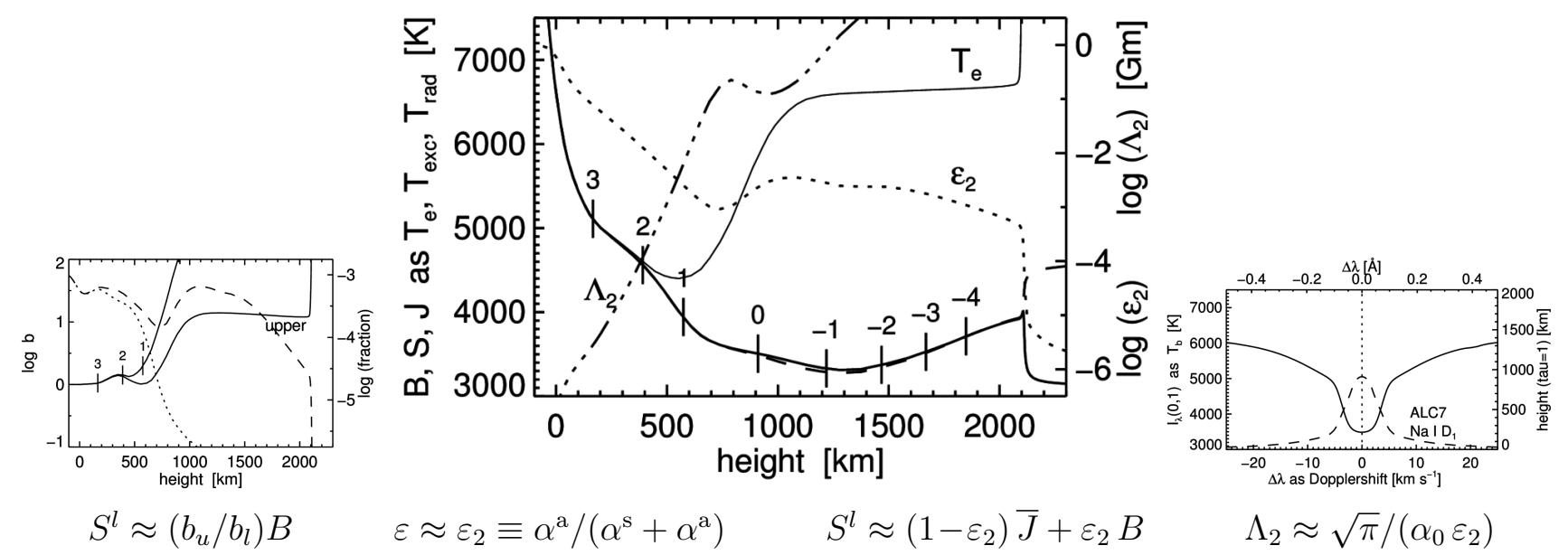

- thin solid: $B_{\lambda_{0}}$ as temperature $T_{\mathrm{e}}$ to remove Planck function variation with wavelength for comparison with other lines. The ALC7 atmosphere has a near-isothermal chromosphere.

- thick solid: NaI $\mathrm{D}_{1} S^{l}$ as formal excitation temperature $T_{\text {exc }}$. The $B>S$ divergence corresponds to the $b_{l}>b_{u}$ divergence in the populations plot, but not equally in their plotted logarithms due to the $B$ and $S$ conversions to formal temperature. The $\log \tau$ ticks are for line center. This scattering line does not sense the ALC7 chromosphere in $S^{l}$.

- dashed: profile-averaged angle-averaged intensity $\bar{J}$ as formal radiation temperature $T_{\text {rad }}$.

- dotted: 2-level photon destruction probability $\varepsilon_{2}$ for the Doppler core. Scale to the right. Follows $N_{e}$, so fairly constant over $1000-2000 \mathrm{~km}$ from increasing hydrogen ionization.

- dot-dashed: 2-level thermalization length $\Lambda_{2}$ for the Doppler core in gigameter. Scale to the right. Example: $\Lambda_{2}=-6$ implies thermalization of $S$ to $B$ at the center of a 2-km thick feature. The curve label is placed near the line-core thermalization height in the mid photosphere. 


\section{LINES FROM THE ALC7 ATMOSPHERE: PROFILE PLOT (Na I D $\left.{ }_{1}\right)$}
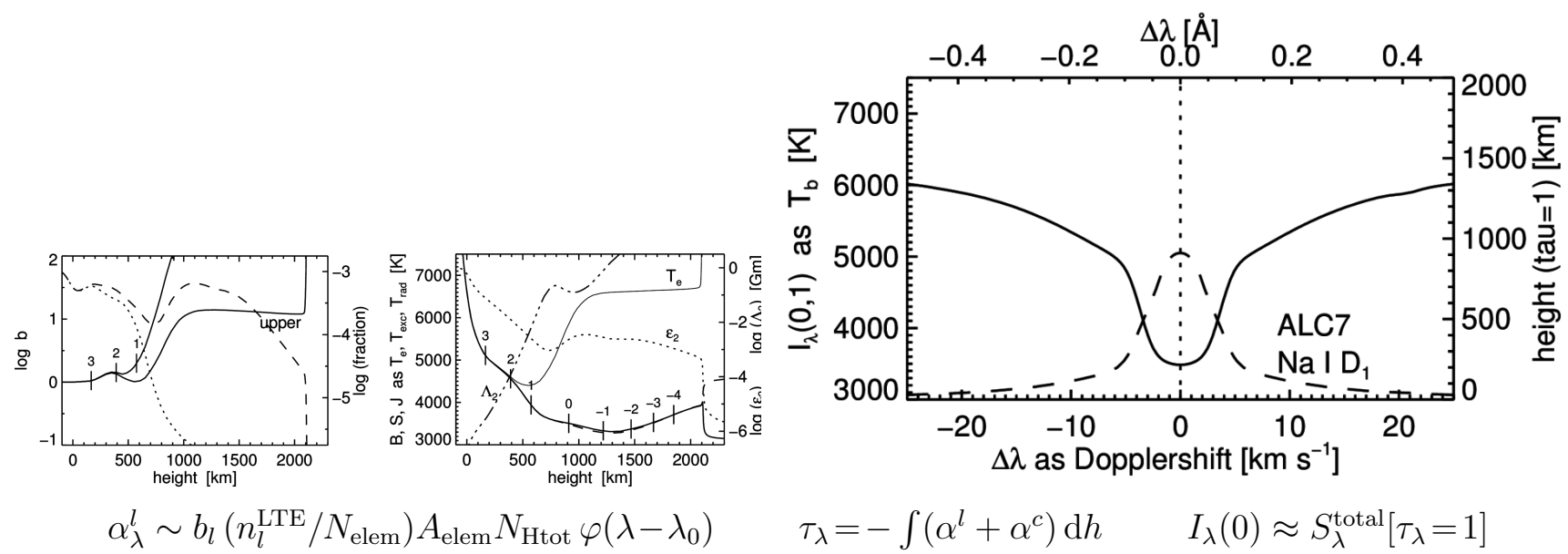

- solid: emergent intensity in the radial direction, represented as formal brightness temperature for comparison with other lines and the Eddington-Barbier estimate ( $B S J$ plot, temperature axes match in the coming line-formation displays). Similarly, the bottom scale for wavelength separation from line center is in $\mathrm{km} \mathrm{s}^{-1}$ for comparison with other lines. Wavelength separations in $\AA$ along the top.

- dashed: $\tau_{\lambda}=1$ height, scale at right.

- dotted, vertical: sampling wavelength(s) for $S$ and $J$ in the $B S J$ plot. Only one for CRD lines (as $\mathrm{Na} I \mathrm{D}_{1}$ ) with frequency-independent line source functions (and $\bar{J}$ in the $B S J$ plot).

- one might overplot an observed solar disk-center profile, but this is misleading because even a perfect match does not imply that the ALC7 model is correct. ALC7 is an idealized didactic star not like the Sun with an easier-to-understand solar-lookalike spectrum. 


\section{Mg I4571 Å FROM THE ALC7 ATMOSPHERE}
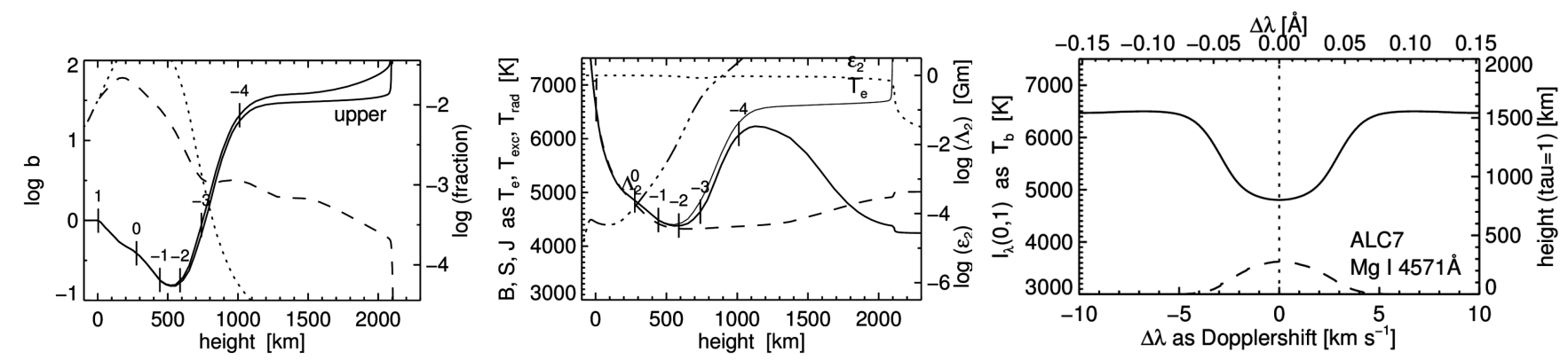

unique photospheric line with LTE source function

- extinction severely out of LTE. Deep $b_{l}$ dip across the ALC7 photosphere from overionization by deeply escaping bound-free scattering ultraviolet radiation, including edges of $\mathrm{Mgl}$ itself at 2512 and $1621 \AA$. Corresponding steep $b_{l}$ rise above $700 \mathrm{~km}$ from ultraviolet underionization where the temperature increases in excess of the ultraviolet radiation temperature.

- this pattern is common to all lines of minority neutrals with ultraviolet ionization wavelenghs, including the electron donors (Mg I, Fe I, Si I, Al I).

- source function unusually close to LTE because this is a "forbidden" intersystem line with small $A_{u l}=2.710^{2} \mathrm{~s}^{-1}$, dominated by collisions $(\varepsilon \approx 1)$ with $b_{u} \approx b_{l}, S^{l} \approx B$ to large heights.

- yet fairly strong because its lower level is the $\mathrm{Mg}$ I ground state

- usefulness: photospheric thermometer but requires ultraviolet NLTE for optical depth 


\section{Fel6301.5 A FROM THE ALC7 ATMOSPHERE}

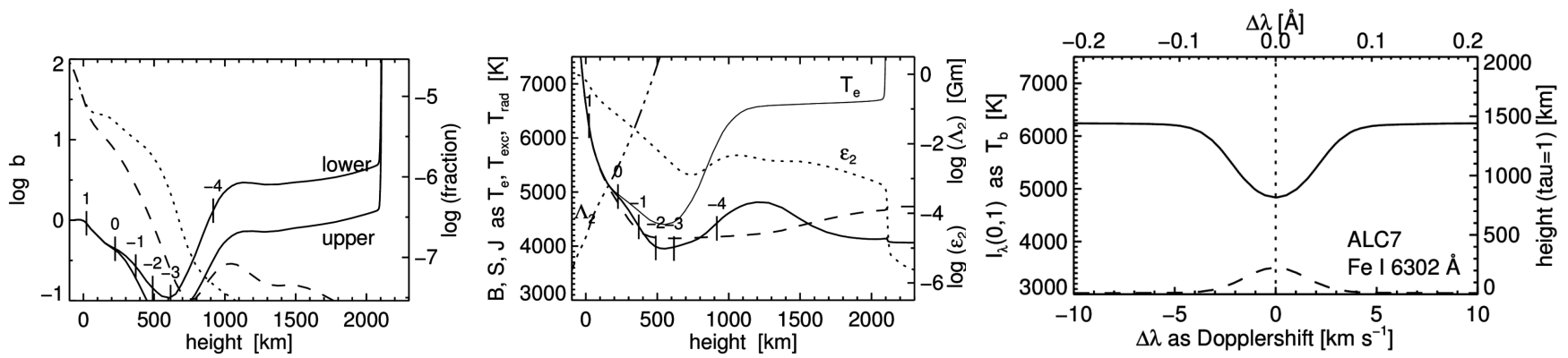

standard polarimetry line

- severe extinction NLTE across the photosphere due to ultraviolet bound-free scattering overionization and affecting the tau scaling ( $b_{l}$ curve)

- minor $S^{l}$ NLTE from resonance scattering in the upper photosphere ( $S<B$ split)

- "inversion" codes (numerical best-fit iteration) sometimes include $S^{l}$ NLTE but usually not extinction NLTE, ignoring that bound-free scattering with $S^{\mathrm{UV}} \approx \bar{J}^{\mathrm{UV}}$ depends on 3D temperature gradients in deeper layers and makes $b_{l}$ (hence $n^{l}$ and $\alpha^{l}$ ) non-local both in space and wavelength

- problem: the enormous density of NLTE lines ("haze") in the ultraviolet affecting $J^{\mathrm{UV}}$

- usefulness: differential line-pair polarimetry with its twin Fe $16302.5 \AA$ 


\section{Mglb $25173 \AA$ FROM THE ALC7 ATMOSPHERE}

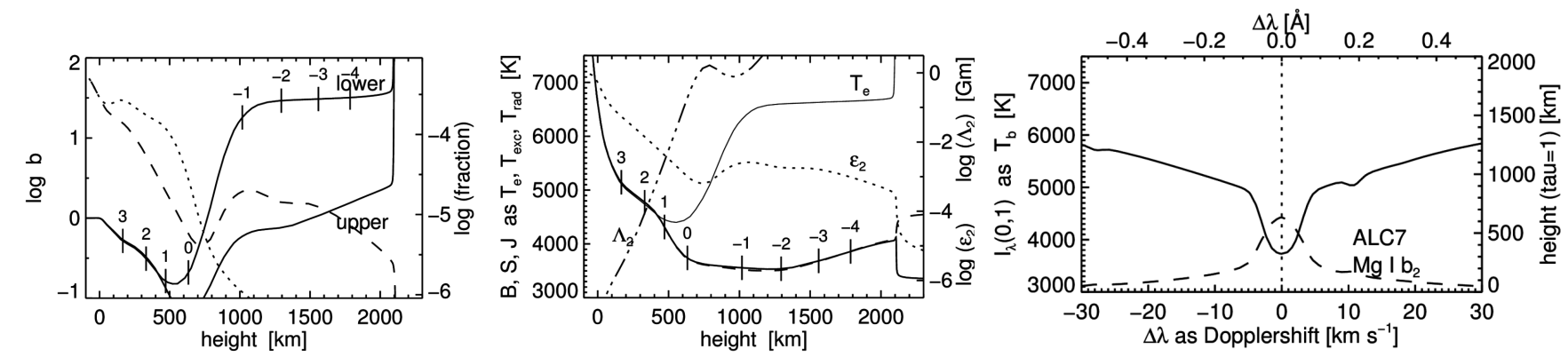

diagnostic of upper photosphere

- large NLTE $n_{l}$ depletion from ultraviolet bound-free scattering across the photosphere

- large NLTE $b_{l}$ increase from ultraviolet scattering offsets Saha decline in chromosphere

- CRD scattering source function with $\varepsilon \approx 10^{-3}$

- similar to $\mathrm{Na} / \mathrm{D}_{1}$

- usefulness: as $\mathrm{Na} / \mathrm{D}_{1}$ but wider core = less asymmetry from reversed granulation 


\section{Na I D 5896 A FROM THE ALC7 ATMOSPHERE}

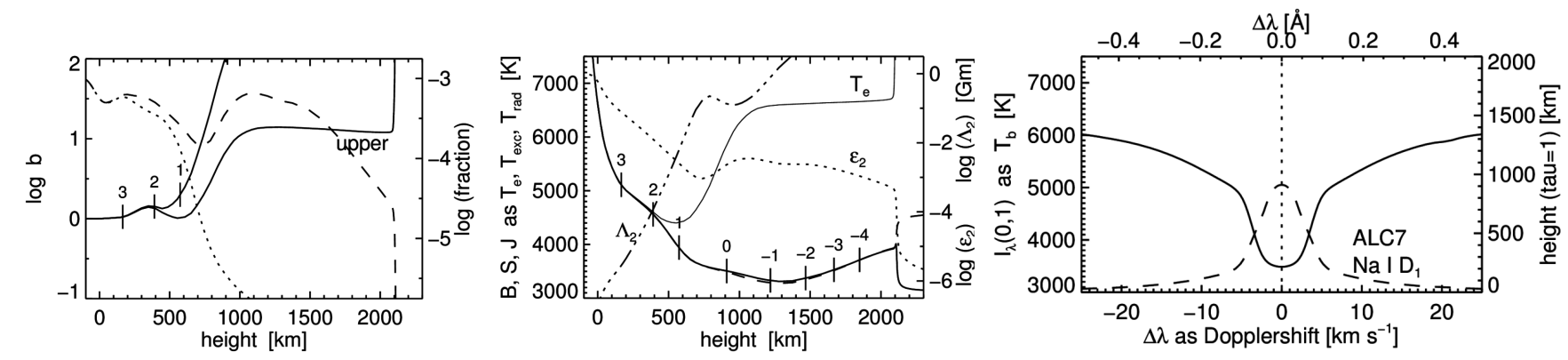

$\mathrm{NaI} D_{1}$ : darkest solar line in optical spectrum = textbook example of two-level scattering

- photon suction offsets ultraviolet overionization across the photosphere

- ultraviolet underionization offsets Saha depletion above $700 \mathrm{~km}$

- 2-level CRD scattering with $\varepsilon \approx 10^{-3}$ and $S \approx \bar{J}<<B$ in the ALC7 chromosphere

- thermalization in mid photosphere: core intensity does not sense ALC7 chromosphere, observed photons are created near the thermalization depth (height of $\Lambda_{2}$ label), observed intensity variation preferentially encodes temperature variation there

- last scattering near $\tau=1$ : Doppler and Stokes inner-wing encoding occurs around $500 \mathrm{~km}$

- usefulness: sharp $\mathrm{Na} / \mathrm{D}_{1}$ Dopplergrams indicate deeply-located shocks in fluxtubes 


\section{Ba II 4554 Å FROM THE ALC7 ATMOSPHERE}

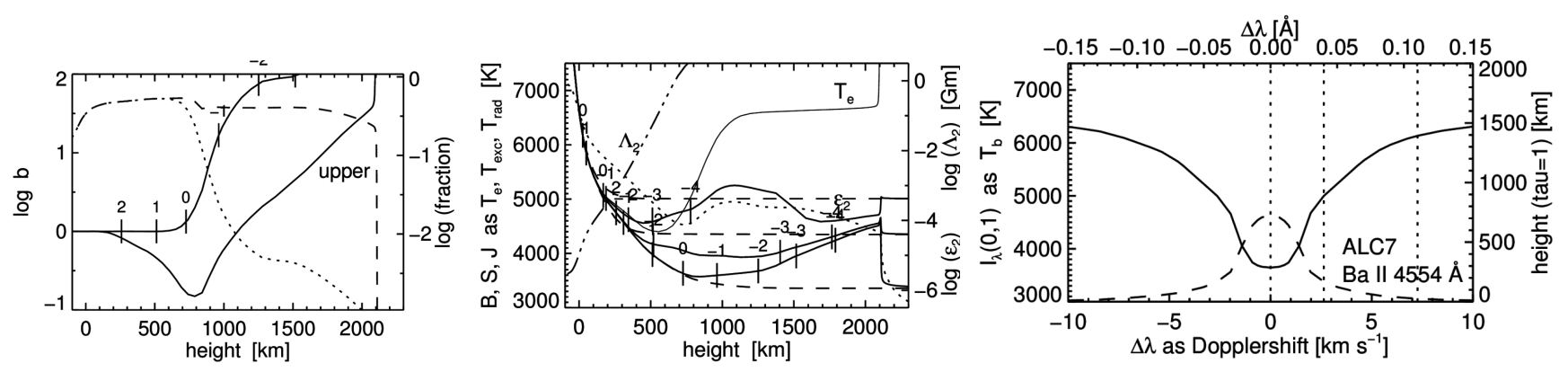

weakest PRD line, best veloctiy diagnostic, good Hanle diagnostic

- extinction LTE up to line-core formation height thanks to photon losses offsetting overionization (edge at $1240 \AA \AA$ outside Ly $\alpha$ )

- steep $b_{l}$ increase above $800 \mathrm{~km}$ from underionization offsets Saha depletion

- resonance line with Grotrian diagram similar to Call K. PRD scattering source function with $\varepsilon \approx 10^{-4}$, therefore different monochromatic $S_{\lambda}$ and $J_{\lambda}$ curves for line center, inner wings, outer wings

- $S^{l}$ split in upper photosphere produces emission wings at the limb (my 1976 eclipse-expedition PhD thesis)

- usefulness: non-thermal Doppler sensitivity from large mass $\left(\sqrt{m_{\mathrm{Ba}} / m_{\mathrm{H}}}=11.7\right)$ intricate near-limb Hanle profile from hyperfine structure 
15 Call 8542 A FROM THE ALC7 ATMOSPHERE

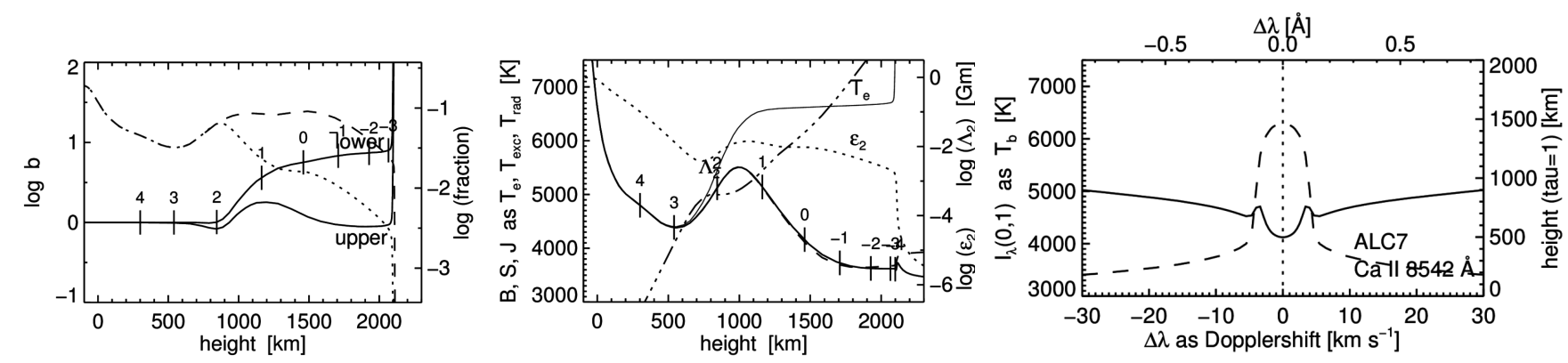

cleanest chromospheric diagnostic in the near infared

- extinction: $b_{l}$ boost from its own photon losses compensates Saha depletion

- $\mathrm{CRD}$ scattering source function with $\varepsilon \approx 10^{-2}$

- core formation spans lower ALC7 chromosphere

- best optical line for chromospheric magnetometry

- usefulness: at longer wavelengths more diffraction but less seeing $\Rightarrow$ prime DKIST line 


\section{Call K 3934 A FROM THE ALC7 ATMOSPHERE}

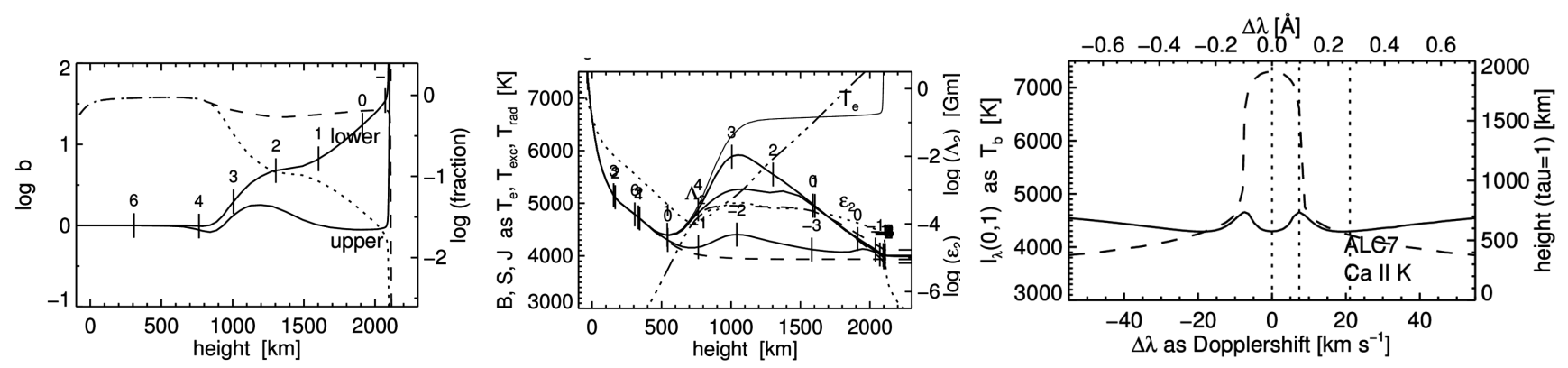

largest extinction in the optical spectrum

- extinction: successive $b_{l}$ boosts from photon losses in infrared triplet and H\&K compensates Saha depletion

- PRD scattering source function with $\varepsilon \approx 10^{-4}$ (split between profile center, peaks, dips)

- core formation spans the ALC7 chromosphere

- narrowness of the Doppler core upsets filter imaging so far

- Sunrise-2/SuFi best so far; high hopes for SST/CHROMIS

- usefulness: best optical chromosphere diagnostic but challenging (bandwidth, $\mathrm{S} / \mathrm{N}$ ) 


\section{Mg II k 2796 A IN THE ALC7 CHROMOSPHERE}
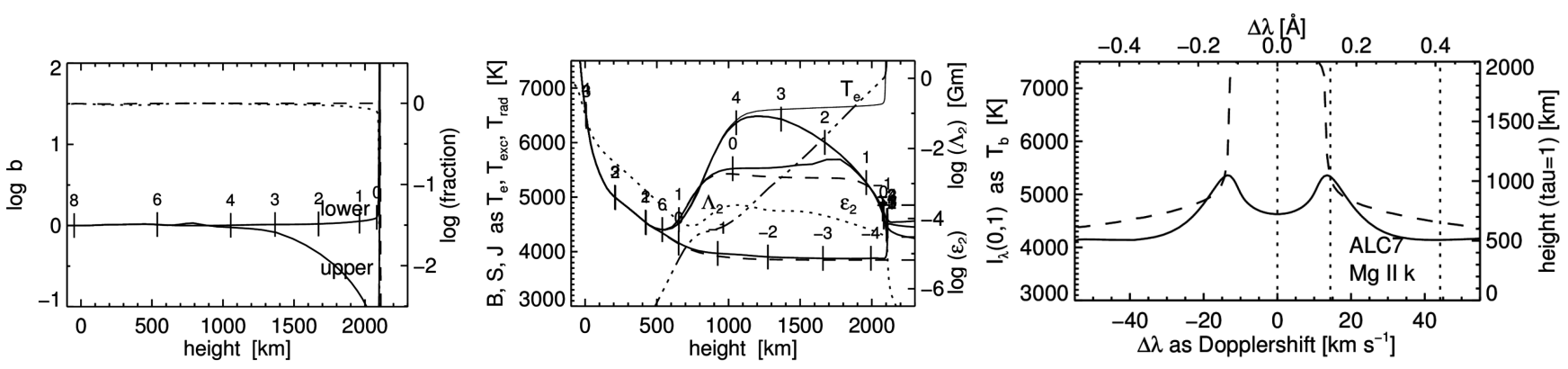

cleanest PRD line and yet larger extincton than Ca II $\mathrm{K}$

- LTE lower-level population and extinction because all Mg sits in the Mg II ground state

- PRD scattering source function with $\varepsilon \approx 10^{-4}$ (split between profile center, peaks, dips)

- textbook scattering decline

- similar to Call K but with $18 \times$ larger abundance and with much darker wings

- usefulness: key diagnostic but requires space platform slitless imaging spectrometry very difficult combine with "triplet" doublet between h \& k (recombination indicator) 


\section{Ly $\alpha 1216 \AA$ IN THE ALC7 CHROMOSPHERE}

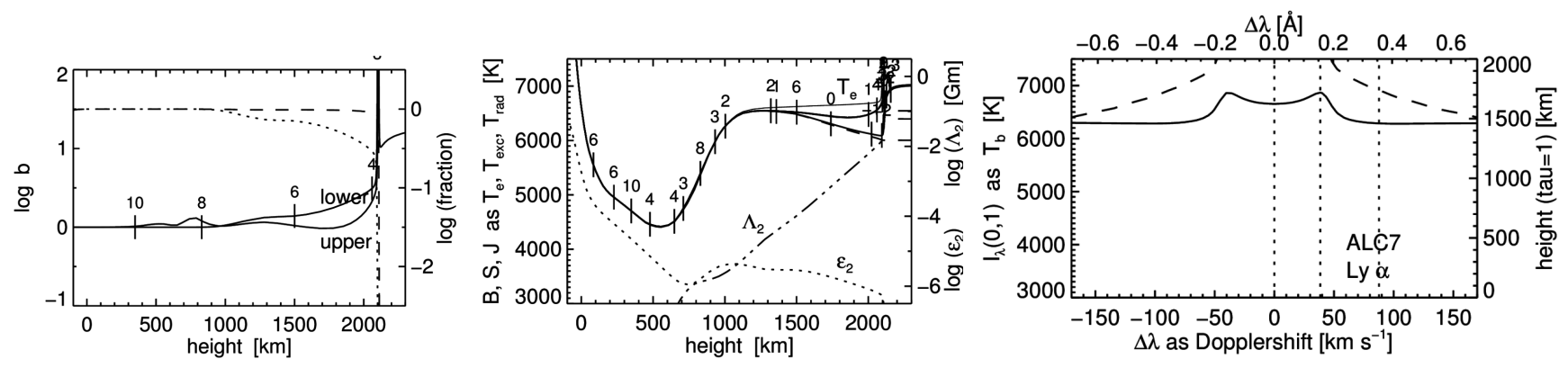

champion: largest extinction and most scattering of all lines

- lower-level population fraction $\approx 1$ : all hydrogen in ground state

- overpopulation of the ground state towards the transition region from photon losses in wings with slight scattering drops $S \approx J<B$

- enormous line-center extinction across the ALC7 chromosphere

- PRD scattering source function with $\varepsilon \approx 10^{-6}$ (split between profile center, peaks, dips)

- $\Lambda$ goes from $\propto 1 / \varepsilon$ towards $\propto 1 / \varepsilon^{2}$ with density from Stark wing development (not shown)

- radiation lock-in from large extinction produces radiative balance $n_{u}\left(A_{u l}+B_{u l} \bar{J}\right)=n_{l} B_{l u} \bar{J}$

- local thermalization from small $\Lambda$ produces $\bar{S} \approx B$ throughout ALC7 chromosphere; $b_{u} \approx b_{l} \approx 1$ implies LTE extinction for $\mathrm{H} \alpha$ where it escapes

- usefulness: premier diagnostic but needs space; slitless imaging spectrometry difficult 


\section{H $\alpha 6563$ A FROM THE ALC7 ATMOSPHERE}

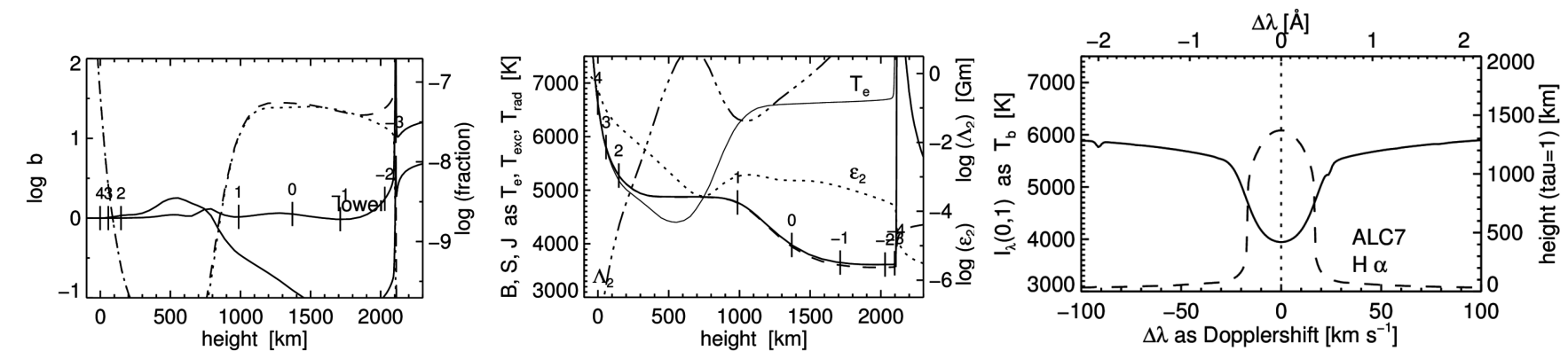

$H_{\alpha}$ : extraordinary from high excitation energy, huge element abundance, on top of Lyo

- lower-level fractional population varies $10^{-10}-10^{-7}$ due to $10 \mathrm{eV}$ in Boltzmann

- extinction coefficient near-LTE up to $2000 \mathrm{~km}$ by Ly $\alpha$ thermalization

- $\mathrm{S}^{l} \approx$ two-level scattering below transition region (few detours, not "photoelectric")

- upper photosphere transparent: core shows fibrils, wings show granules

- Eddington-Barbier tau $=1$ in chromosphere, but photon creation in deep photosphere

- large $\mathrm{J}$ across T-min from backscattering: ALC7 chromosphere $\approx$ scattering attenuator

- wide line core from small atomic mass in Doppler broadening $\sim \sqrt{2 k T / m_{\mathrm{H}}+v_{\text {micro }}^{2}}$

- extended wings from linear Stark effect in deep photosphere (Holtsmark distribution)

- usefulness: prominences, flares, Ellermans, dynamic fibrils, spicules-II, ...=non-E 


\section{CANONICAL CHROMOSPHERIC LINE FORMATION}
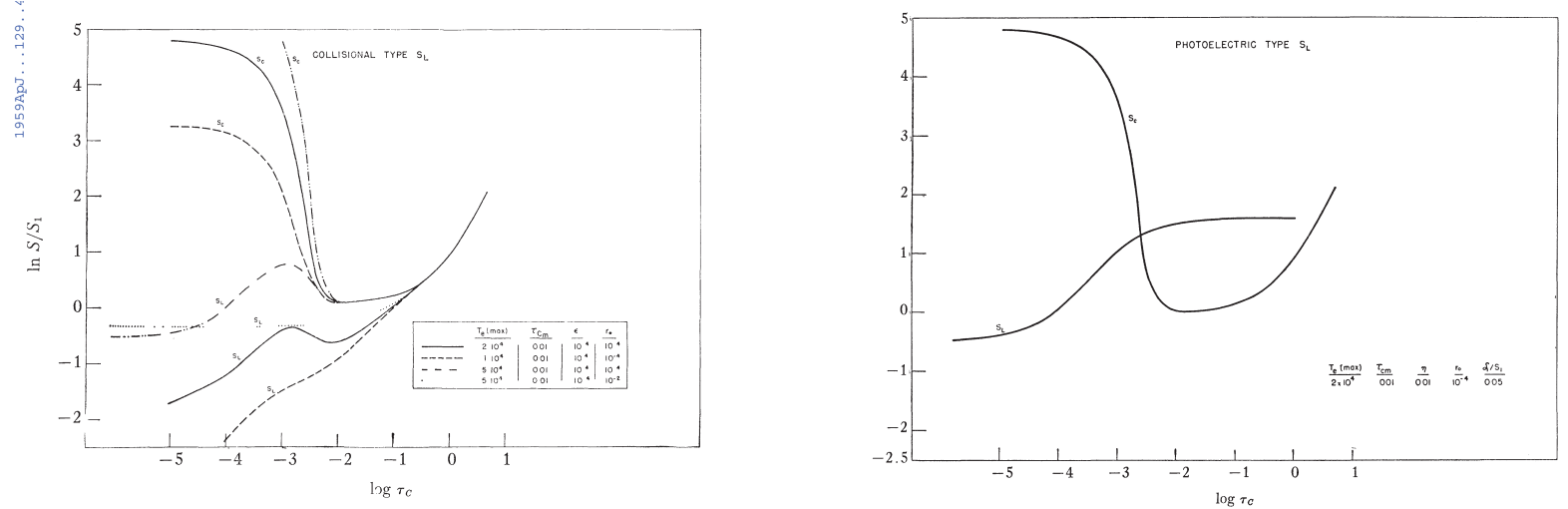

- CRD line source function including detour paths:

$$
\begin{aligned}
S_{\nu_{0}}^{l} & =\frac{\bar{J}_{\nu_{0}}+\varepsilon_{\nu_{0}}^{\prime} B_{\nu_{0}}(T)+\eta_{\nu_{0}}^{\prime} B_{\nu_{0}}\left(T_{\mathrm{d}}\right)}{1+\varepsilon_{\nu_{0}}^{\prime}+\eta_{\nu_{0}}^{\prime}} \\
& =\left(1-\varepsilon_{\nu_{0}}-\eta_{\nu_{0}}\right) \bar{J}_{\nu_{0}}+\varepsilon_{\nu_{0}} B_{\nu_{0}}(T)+\eta_{\nu_{0}} B_{\nu_{0}}\left(T_{\mathrm{d}}\right)
\end{aligned}
$$

- $\varepsilon=$ upper-lower collisional destruction fraction of total extinction $\eta=$ detour-path extinction fraction of total extinction

$\varepsilon^{\prime}, \eta^{\prime}=$ idem as ratio to scattering extinction

$\bar{J}=$ profile-averaged angle-averaged intensity

$T_{\mathrm{d}}=$ formal detour excitation temperature: $\left(g_{u} D_{u l}\right) /\left(g_{l} D_{l u}\right) \equiv \exp \left(h \nu_{0} / k T_{\mathrm{d}}\right)$

- line source function split (Thomas 1957ApJ...125..260T):

"collision type" (H\& K) or "photoelectric type" ( $\mathrm{H} \alpha$, Balmer continuum feeding) 


\section{$21 \mathrm{H} \alpha$ SOURCE FUNCTION IN THE ALC7 CHROMOSPHERE}

after Rutten \& Uitenbroek 2012A\&A...540A..86R
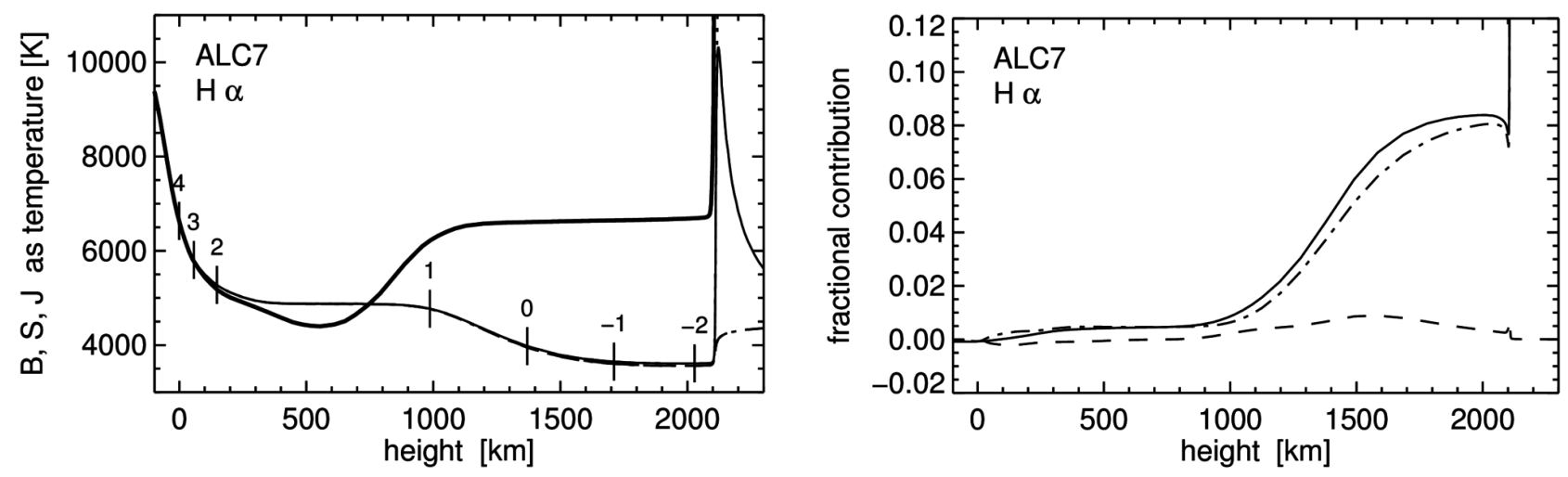

- $S_{\nu_{0}}^{l}=\left(1-\varepsilon_{\nu_{0}}-\eta_{\nu_{0}}\right) \bar{J}_{\nu_{0}}+\varepsilon_{\nu_{0}} B_{\nu_{0}}(T)+\eta_{\nu_{0}} B_{\nu_{0}}\left(T_{\mathrm{d}}\right)=\bar{J}_{\nu_{0}}+\varepsilon_{\nu_{0}}\left[B_{\nu_{0}}(T)-\bar{J}_{\nu_{0}}\right]+\eta_{\nu_{0}}\left[B_{\nu_{0}}\left(T_{\mathrm{d}}\right)-\bar{J}_{\nu_{0}}\right]$

The detour part $\eta_{\nu_{0}}\left[B_{\nu_{0}}\left(T_{\mathrm{d}}\right)-\bar{J}_{\nu_{0}}\right] / S_{\nu_{0}}^{l}$ exceeds the collision part $\varepsilon_{\nu_{0}}\left[B_{\nu_{0}}(T)-\bar{J}_{\nu_{0}}\right] / S_{\nu_{0}}^{l}$. However, their sum $\left[S_{\nu_{0}}^{l}-\bar{J}_{\nu_{0}}\right] / S_{\nu_{0}}^{l}$ (solid) reaches only a few percent so $S_{\nu_{0}}^{l} \approx \bar{J}_{\nu_{0}}$. Across the ALC7 chromosphere $\mathrm{H} \alpha$ is a scattering line, not "photoelectrically controlled".

- The $\mathrm{H} \alpha$ core is dominated by resonance scattering with a formation gap below the chromosphere filled by backscattered radiation. The ALC7 chromosphere acts as scattering attenuator building up its own irradiation. Most emerging photons are created in the deep photosphere where $\varepsilon_{\nu_{0}} \approx 1$ and $\bar{J}_{\nu_{0}} \approx B_{\nu_{0}}(T)$. The granulation pattern has larger contrast than the fibril pattern but is washed out in the scattering across the gap.

- The ALC7 $\mathrm{H} \alpha$ core formation is well described by the Eddington-Barbier approximation for an irradiated finite isothermal scattering atmosphere. 


\section{NON-EQUILIBRIUM HYDROGEN IONIZATION IN 1D SHOCKS}

Carlsson \& Stein 2002ApJ...572..626C

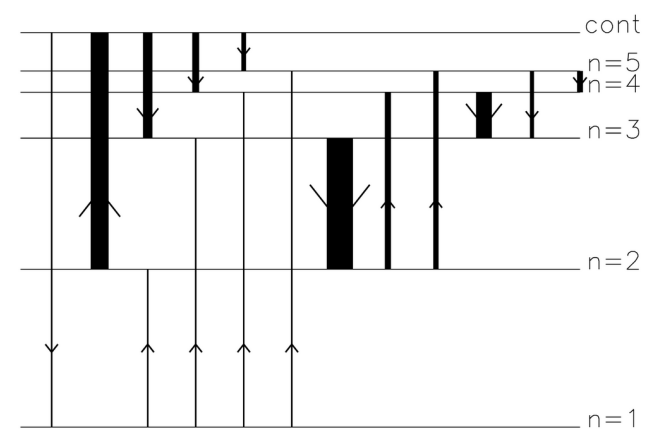

- RADYN code: 1D(t) hydrodynamics, time-dependent, NLTE radiation, simple PRD

- observed subphotosphere piston drives acoustic waves up that shock near $h=1000 \mathrm{~km}$

- Ly $\alpha$ scatters in radiative balance and controls $n=2$. Within shocks $S \approx J$ saturates to $B$ from radiation lock-in (increased $\varepsilon$ from partial hydrogen ionization) so that $b_{2} \approx 1$

- collisional Ly $\alpha$ balancing has Boltzmann temperature sensitivity: fast (seconds) in hot gas, slow (minutes) in cool gas, resulting in retardation: post-shock cooling gas maintains the high $n_{2}$ shock value at increasing $b_{2}$ during minutes, up to huge overpopulation $\left(b_{2} \approx 10^{10}\right)$

- ionization from $n=2$ in the $3.4 \mathrm{eV}$ alkali-like hydrogen top is an instantaneous statisticalequilibrium balance driven by Balmer continuum $J \neq B$ and photon losses in Balmer and higher lines, with $b_{\text {cont }} / b_{2} \approx 10^{-1}$ in hot and $\approx 10^{+3}$ in cool gas, adding to the retarded $b_{2}$

- between shocks hydrogen remains hugely overionized versus SE and LTE predictions 


\section{NON-E HYDROGEN IONIZATION IN 2D MHD SHOCKS}

Leenaarts et al. 2007A\&A...473..625L
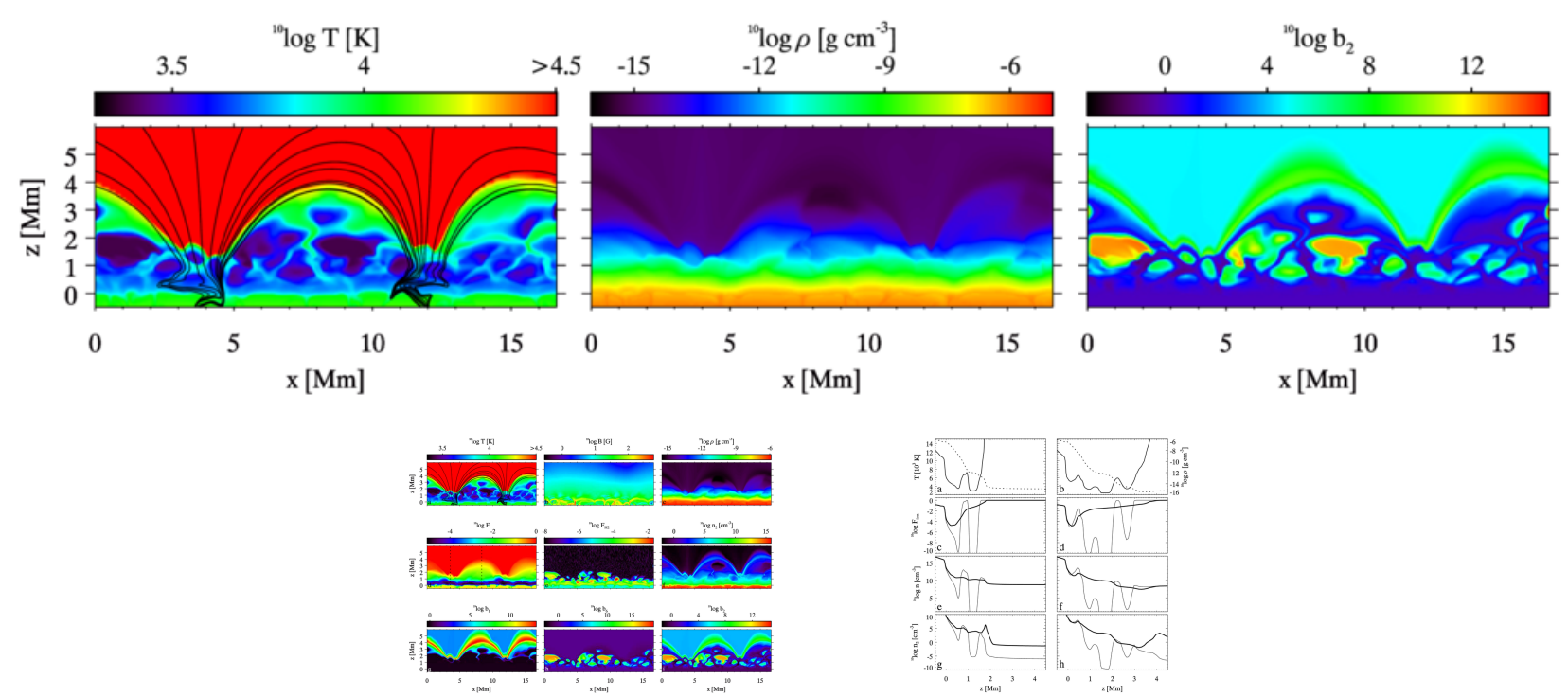

- in shocks Ly $\alpha$ has $S \approx B$ from high $T$ (fast balancing) and $N_{e}(10 \% \mathrm{H}$ ionization)

- retarded collisional balancing in Ly $\alpha: n_{2}$ hangs near high shock value $n_{2} \approx n_{2}^{\mathrm{LTE}}$

- gigantic post-shock $n=2$ overpopulations versus LTE ("S-B underestimates")

- yet larger post-shock overionization from hydrogen-top Balmer balancing

- approximation: Lyman lines in radiative balance $\Rightarrow$ green arches artifacts 


\section{$24 \mathrm{H} \alpha$ IN BIFROST}

1D plane-parallel: Rutten \& Uitenbroek 2012A\&A...540A..86R
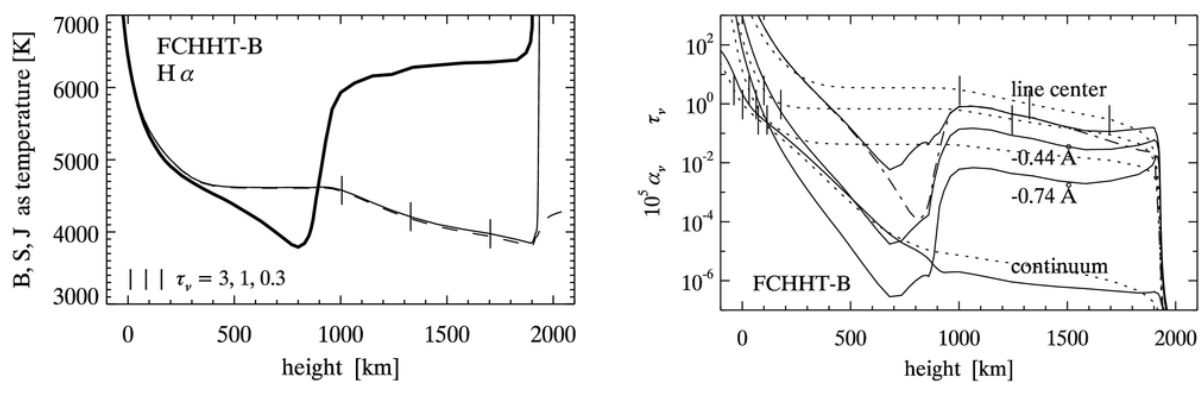

3D non-E MHD snapshot: Leenaarts et al. 2012ApJ...749...136L
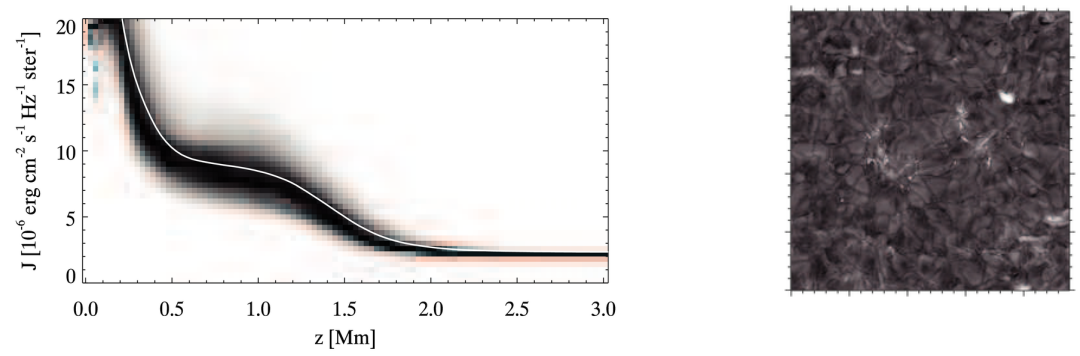

- $\mathrm{H} \alpha$ is a pure scattering line with $S \approx J$ and a deep opacity dip in the upper photosphere

- 3D scattering across the opacity gap enhances fibril visibility

- core darkness measures density, core width measures temperature

- caveat: BIFROST snapshot, no non-E line synthesis $\Rightarrow \mathrm{H} \alpha$ severely underestimated 


\section{OSLO SIMULATION VERSUS 1D STANDARD MODELS}
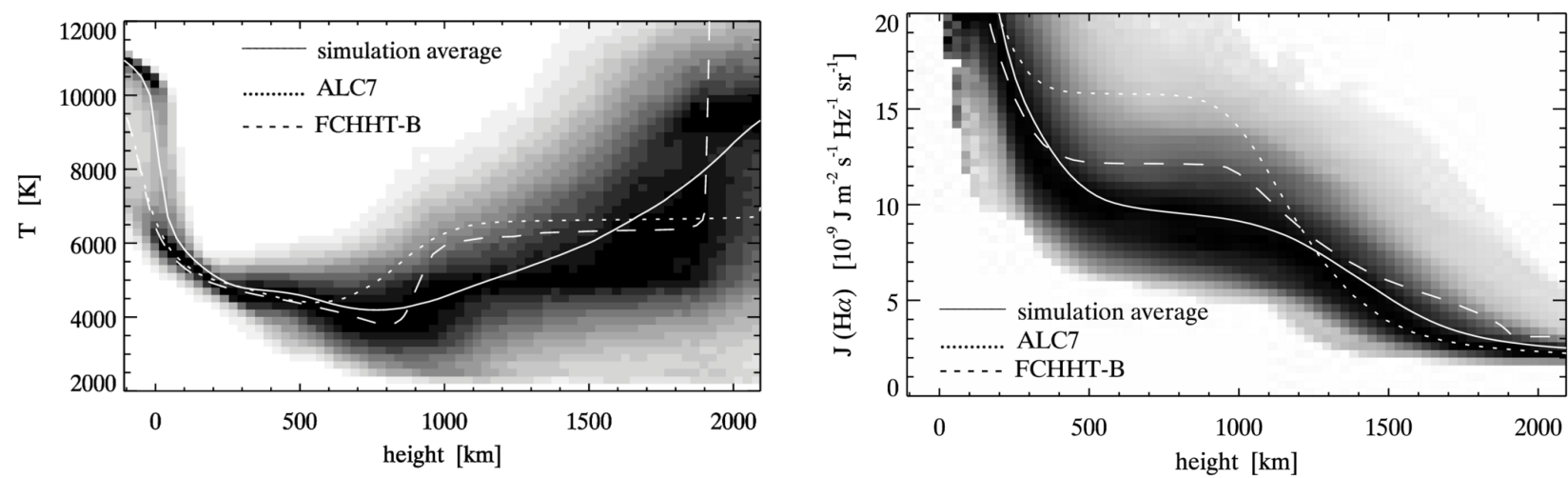

- simulation = state-of-the-art: $3 \mathrm{D}(\mathrm{t}), \vec{B}$, non-HE, SE populations but NE for $\mathrm{H}$ Leenaarts, Carlsson \& Rouppe van der Voort 2012ApJ...749..136L

- $\mathrm{ALC7}=\mathrm{UV}$ fit: 1D static, no $\vec{B}, \mathrm{HE}+$ microturbulence, SE populations Avrett \& Loeser 2008ApJS..175..229A

- FCHHT-B = UV fit: 1D static, no $\vec{B}, \mathrm{HE}+$ imposed acceleration, SE populations Fontenla, Curdt, Haberreiter, Harder \& Tian 2009ApJ...707..482F

The $T$ and $J_{\nu}(\mathrm{H} \alpha)$ behavior seems arguably similar. However, the conceptual differences between plane-parallel static hydrostatic-equilibrium modeling and the $3 \mathrm{D}(\mathrm{t}) \mathrm{MHD}$ simulation are enormous (cf. Newtonian gravitation versus general relativity). The $T(h)$ stratifications in the simulation vary tremendously, with shocks propagating upwards and sideways and the increase to coronal temperature dancing up and down over a large height range. 


\section{HYDROGEN AUREOLE BOOSTING IN COOL GAS BESIDE HOT GAS}

Fontenla et al. 2009ApJ...707..482F Rutten \& Uitenbroek 2012A\&A...540A..86R Rutten 2016A\&A...590A.124R
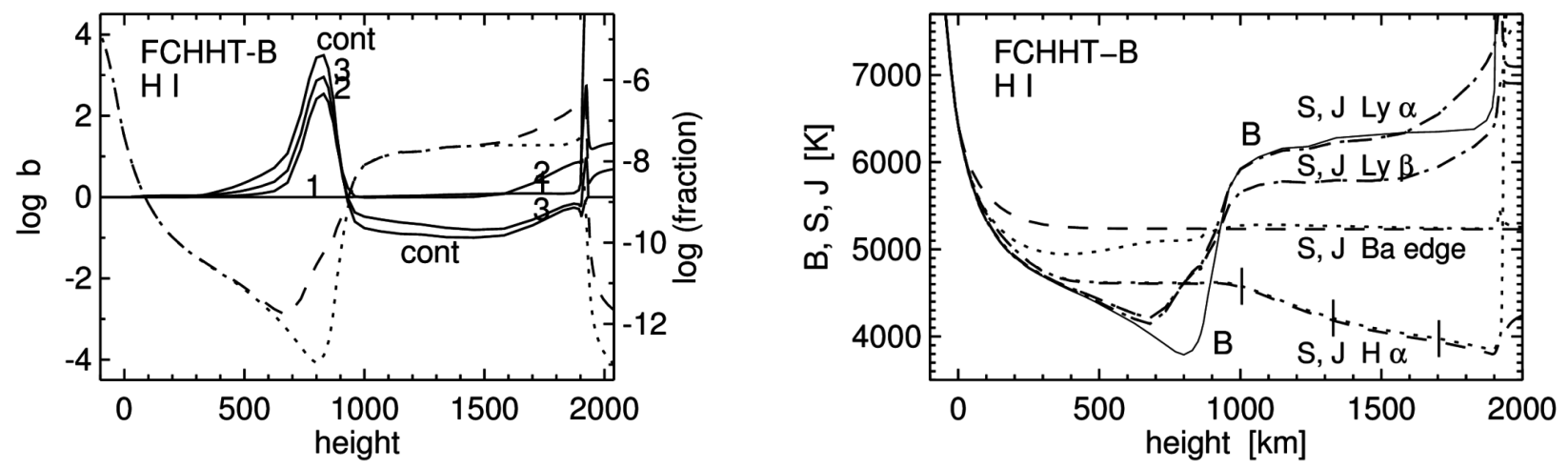

FCHHT-B: steep $B$ rises to chromosphere and corona emulate adjacent cool and hot features

$\operatorname{Ly} \alpha$ : scattering back-radiation boosts $S_{\mathrm{Ly} \alpha} \approx J_{\mathrm{Ly} \alpha}$ and $\mathrm{H} \alpha$ extinction $\propto b_{2} \approx S_{\mathrm{Ly} \alpha} / B_{\mathrm{Ly} \alpha}$ towards hot-feature value (left: dotted $n_{2}^{\mathrm{LTE}} / N_{\mathrm{Htot}}$, dashed actual $n_{2} / N_{\mathrm{Htot}}$ )

ionization: also $b_{2}$-boosted, with additional $b_{\text {cont }} / b_{2}$ offset defined by the Balmer continuum

Ly $\beta$ : $b_{3}$ between $b_{2}$ and $b_{\text {cont }}$ and sharing $\mathrm{H} \alpha$ photon losses

$\mathrm{H} \alpha$ : the FCHHT-B chromosphere is a back-scattering attenuator just as in the ALC7 atmosphere. The $b_{2}$ peak from $\mathrm{Ly} \alpha$ irradiation does not affect $\mathrm{H} \alpha$ because even with this boost the $\mathrm{H} \alpha$ extinction in the temperature miminum remains negligible.

A hot feature embedded in cooler gas has a similar Ly $\alpha$ scattering aureole enhancing $\mathrm{H}$ ionization and $\mathrm{H} \alpha$ extinction around it. A temporary hot disturbance leaves such spread-out boost behind (a wake when moving). 


\section{$27 \mathrm{H} \alpha$ EXTINCTION RECIPE}

- retarded Ly $\alpha$ balancing: extinction memory of hot moments

- $n_{2} \approx n_{2}^{\mathrm{LTE}}$ in hot shocks from fast Ly $\alpha$ balancing and increased $\varepsilon$

- $n_{2}$ decays slowly, tracking high shock values

- gigantic $b_{2}$ in post-shock cooling clouds until next shock

- Lya scattering: aureole boosting

- Ly $\alpha$ scattering defines $S_{\mathrm{Ly}} \approx J_{\mathrm{Ly}}$ with radiative balance

- hot features in cool gas have Ly $\alpha$ scattering aureoles

- HI top ( $n \geq 2$ including $n_{\text {ion }}$ ) boosted in aureoles

- Ho extinction recipe

- find hottest instance nearby $(\sim 300 \mathrm{~km})$ and in recent past ( $\sim$ minutes)

- compute Saha-Boltzmann fractional $n=2$ population then and there

- use this extinction value in cooler gas around it and afterwards

- small hot features leave wider $\mathrm{H} \alpha$ marks (as the grin of the Cheshire cat)

- fast small hot features leave wider $\mathrm{H} \alpha$ trails (as contrails from jet engines)
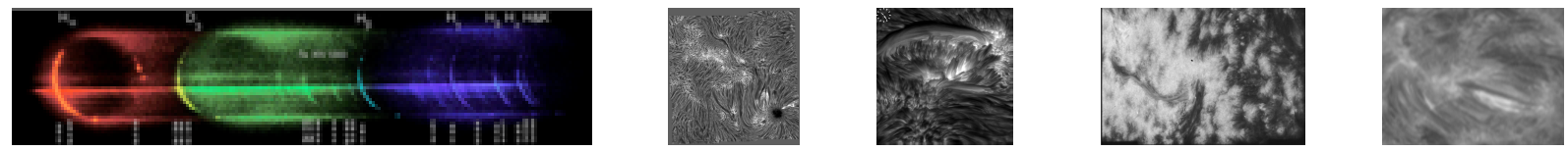


\section{$28 \mathrm{H} \alpha$ HOT-ONSET EXTINCTION}
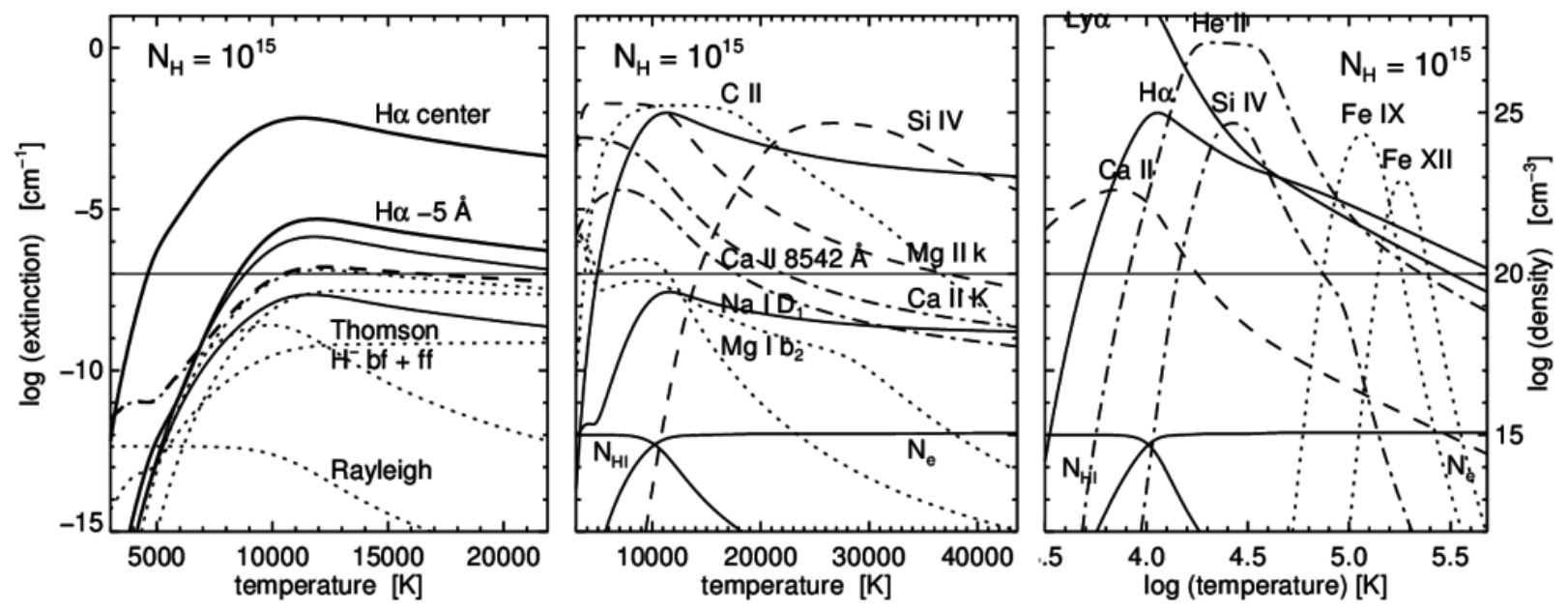

- bachelor exercise "Cecilia Payne": compute $N_{\mathrm{e}}$ and $\kappa^{\mathrm{LTE}}$ for given $N_{\mathrm{H}}$

- line at $\log (\alpha)=-7: \tau=1$ across $100-\mathrm{km}$ slab

- $\mathrm{H} \alpha$ moustaches beyond continuum: linear Stark + Holtsmark

- photosphere: $\mathrm{H} \alpha$ much weaker than Call H \& K, Ly $\alpha \mathrm{mm}$ photon paths

- chromosphere: $\mathrm{H} \alpha \approx \mathrm{Ca}$ ll $8542 \AA$, opposite temperature sensitivity

- clapotispheric shocks: $\mathrm{H} \alpha \sim \mathrm{Ca}$ II $\mathrm{H} \& \mathrm{~K}$ and remembers that

- outburst onsets: extraordinary $\mathrm{H} \alpha$ opacity, competing with (E)UV lines 